\title{
Comparative analysis of the SPL genes among four Ipomoea species and the evidence for a role of some IbSPLs in storage root development in sweet potato (Ipomoea batatas)
}

Haoyun Sun

Jiangsu Normal University

Jingzhao Mei

Tianjin Medical University

Wenqian Hou

Jiangsu Normal University

Yang Zhang

Jiangsu Normal University

Tao Xu

Jiangsu Normal University

Shaoyuan Wu

Jiangsu Normal University

Lei Zhang ( $\square$ leizhang@jsnu.edu.cn )

Jiangsu Normal University

\section{Research Article}

Keywords: Ipomoea, SPL transcription factor, evolutionary patterns, root development, expression profiles

Posted Date: August 12th, 2021

DOl: https://doi.org/10.21203/rs.3.rs-654042/v1

License: () (i) This work is licensed under a Creative Commons Attribution 4.0 International License. Read Full License 


\section{Abstract \\ Background}

As a major family of plant-specific transcription factors, SQUAMOSA PROMOTER BINDING PROTEIN-LIKE (SPL) genes play crucial regulatory roles in plant growth, development, and stress tolerance. SPL transcription factor family has been widely studied in various plant species, however, there are no systematic studies on SPL genes in genus Ipomoea.

\section{Results}

In this study, a total of $29,27,26,23$ SPL genes were identified in Ipomoea batatas, Ipomoea trifida, Ipomoea triloba, and Ipomoea nil, respectively. Phylogenetic analysis indicated that Ipomoea SPL genes could be clustered into eight clades. SPL members within the same clade showed similar gene structures, domain organizations, and cis-acting element compositions, suggesting similarity of biological function potentially. Evolutionary analysis revealed that segmental duplication events played a major role in the Ipomoea genus-specific expansion of SPL genes. Of these Ipomoea SPL genes, 69 were predicted as the target genes of miR156, and $7 \mathrm{IbSPL}$ genes were further confirmed by degradome data. Additionally, IbSPL genes showed diverse expression patterns in various tissues, implying their functional conservation and divergence. Finally, by combining the information from expression patterns and regulatory sub-networks, we found that four IbSPL genes (IbSPL16/IbSPL17/IbSPL21/IbSPL28) may be involved in the formation and development of storage roots.

\section{Conclusions}

This study not only provides novel insights into the evolutionary and functional divergence of the $S P L$ genes in all available sequenced species in genus Ipomoea, but also lays a foundation for further elucidation of the potential functional roles of $I b S P L$ genes during storage root development.

\section{Background}

The SQUAMOSA promoter-binding protein-like ( $S P L)$ gene family is one of the plant-specific transcription factor families, which play fundamental roles in plant growth, development, and stress tolerance [1-5]. The most common feature of SPL gene members is that they all contain SQUAMOSA promoter-binding (SBP) domain, which was composed of three distinct motifs: two non-interleaved zinc-binding sites (CysCys-Cys-His and Cys-Cys-His-Cys), and one nuclear localization signal (NLS) at the C-terminus [1]. SPL genes ( $A m S B P 1$ and AmSBP2) were first discovered in Antirrhinum majus based on their functions on flower development [6]. Since then, numerous homologs have been identified and characterized in model species, such as Arabidopsis thaliana [7], Oryza sativa [8], and Solanum lycopersicum [9]. With the 
increasing number of sequenced genomes, more and more SPL members have been annotated and reported in non-model plants, such as apple [10], pepper [11], soybean [12], and so on.

The functions of $S P L$ genes in model plants have been well studied, and showed functional divergence. In A. thaliana, AtSPL3, AtSPL9 and AtSPL10 have been demonstrated to regulate root development $[13,14]$. Meanwhile, similar roles have been reported in rice [15] and apple [16]. Other studies have found that SPL proteins participate in vegetative and reproductive phase transitions. For instance, AtSPL3/4/5 promote flowering by directly activating the expression of three flowering integrator genes namely, AP1, FUL and LFY [17]. In addition, SPL proteins have also been found to participate in fruit development and grain yield. For example, LeSPL-CNR regulates cell wall disassembly and carotenoid biosynthesis during fruit ripening in tomato [18, 19]; overexpression of OSSPL 16 promotes cell division and grain filling, with positive consequences for grain width and yield in rice [20]. SPL genes are also known as the targets of miR156, thus forming a functional regulatory network of miR156-SPL [2]. In the miR156-SPL module, AtSPL9 has a negative effect on anthocyanin accumulation [21]; OsSPL7 enhances disease resistance against bacterial blight [22]. The functions of $S P L$ genes have been deeply studied in Arabidopsis and other model plants, however, studies on SPL genes in genus Ipomoea are relatively scarce.

The genus Ipomoea, which includes 500-600 species, possesses the largest number of species in the family Convolvulaceae [23]. Species in genus Ipomoea are widely distributed in the world, which are of great value in the fields of industry and agriculture [23-25]. For example, Japanese morning glory, Ipomoea nil (L.) Roth. $(2 \mathrm{n}=2 \mathrm{x}=30)$, is cultivated as an ornamental plant due to its various patterns of flower color [24]. Sweet potato, Ipomoea batatas (L.) Lam. $(2 n=6 x=90)$, is ranked as the seventh most important crop globally due to its strong adaptability, stable yield and high nutritional value [25]. The storage roots of sweet potato, which are mainly harvested, are of great significance to the nutrient content and yield [26]. Up to now, the genomes of four species (I. batatas, I. trifida, I. triloba and I. nil) have been sequenced in genus Ipomoea [27-29]. Among these species, I. trifida is the most closely related diploid to I. batatas, followed by I. triloba and I. nil [29]. The reported haplotype-resolved genome assembly of $I$. batatas is of low-quality, making it difficult to accurately identify and characterize genes. By contrast, the genome assembly of the other three diploid relatives are of high-quality, and can be used as robust references for I. batatas. All in all, the availability of these genomes makes it possible to perform genomewide comparative analysis of SPL genes in genus Ipomoea.

In this study, we first conducted the genome-wide identification and characterization of SPL genes in four Ipomoea species, including I. batatas, I. trifida, I. triloba and I. nil. We next investigated phylogenetic relationship and evolutionary patterns of $S P L$ genes in these four species. We then determined the expression patterns of IbSPL genes using transcriptome and qRT-PCR in different tissues or under various hormones treatments. Finally, we performed the WGCNA to construct the co-expression network, and inferred the putative functions for the $I b S P L$ genes in the storage root of sweet potato. Our work will not only provide insights into the evolutionary conservation and diversification of the $S P L$ genes in genus Ipomoea, but also lays the foundation for further research on the IbSPL genes related to the storage root development in sweet potato. 


\section{Results}

\subsection{Identification of SPL genes in four Ipomoea species}

To obtain SPL genes in Ipomoea species, two methods, BLASTP and HMM, were used for identification, and two online databases, SMART and ScanProsite, were used for confirmation. As a result, a total of 29, 27, 26, 23 SPL genes were identified in I. batatas (Ib), I. trifida (Itf), I. triloba (Itb), and I. nil (In), respectively. For consistency, the Ipomoea SPL genes were renamed according to their chromosomal location order in each genome (Supplementary Table S1). The numbers of SPL genes and their percentage of the total genes in each species were displayed (Figure 1a). We found that $I$. nil genome has the least number of $S P L$ genes compared to the other three species.

Subcellular localization analysis showed that most of the Ipomoea SPL proteins ( 94 , 89.52\%) were localized in the nucleus (Supplementary Table S1), suggesting their critical role in regulatory functions. Besides, the physical and chemical properties of SPL proteins were significantly different within species, but exhibited similar patterns among the four species (Figure 1b-d, Supplementary Table S1). To be more specific for all of the Ipomoea SPL proteins, the number of amino acids ranged from 103 (InSPL9) to 1141 (IbSPL 11), the molecular weight (MWs) varied from 12.01 (InSPL9) to 126.51 (IbSPL 11) kDa, the isoelectric points (pls) ranged from 5.40 (ItfSPL 14) to 10.55 (InSPL 15).

\subsection{Comparative phylogenetic analysis of Ipomoea SPL genes}

To explore the evolutionary relationship among the Ipomoea SPL genes, a rooted neighbor-joining phylogenetic tree was constructed using 105 SPL proteins from four Ipomoea species and 51 SPL proteins from other five plant species (A. thaliana, S. Iycopersicum, O. sativa and Chlamydomonas reinhardii) (Figure 2, Supplementary Table S2). Based on the classification of SPLs from A. thaliana, S. Iycopersicum and $O$. sativa [7-9], Ipomoea $S P L$ genes were classified into eight clades, which were numbered from I to VIII (Figures 1,2). All clades had at least one SPL gene in each Ipomoea species, indicating the conservation of SPLS across Ipomoea genomes. However, the number of SPLS in certain clades were highly variable among Ipomoea species, suggesting the diversity of SPLS in genus Ipomoea. Clade I was the smallest subfamily, containing only one member for each Ipomoea species. Clade IV contained the highest number of $S P L S(>26 \%$ ) in genus Ipomoea, and it could be further divided into three subclades (IV$a$, IV-b, and IV-c). Members of IV-b and IV-c subclades only contain SPL genes from Ipomoea species, and no homologs of other species were found, which indicates that the Ipomoea SPL genes in these two subclades were generated in an ancient ancestor of the Ipomoea lineage. In addition, the phylogenetic analysis also indicated that most IbSPL genes were closer to ItfSPL genes than either ItbSPL or InSPL genes, supporting the fact that $I$. trifida is the most closely related diploid to hexaploid sweet potato [29]. When the number of SPL genes in Ipomoea species were compared with other species, we found that the number in Ipomoea species (the average number of $S P L$ genes in 4 Ipomoea species was 26) was greatly increased by almost 1.6, 1.4 and 2 times than that in $A$. thaliana (16), O. sativa (19) and $S$. 
lycopersicum (13), respectively. The results indicated that Ipomoea SPL genes have undergone extensive expansion after the speciation of $S$. lycopersicum.

\subsection{Gene and protein structure of the Ipomoea SPL family}

To explore the structural diversity of the Ipomoea SPL genes, intron/exon structure analysis were carried out (Figure 3). Gene structure illustrations showed the high variation in the number of exons for Ipomoea $S P L$ genes, ranging from 2 to 15 (Figure 3a, Supplementary Table S1). For instance, IbSPL20 possesses the largest number (15) of exons, whereas most of the Ipomoea SPL genes in Clade VI contained the least number (2) of exons. Moreover, most Ipomoea SPL genes in the same clade exhibited similar gene structures, even if they belong to different species. For example, $S P L$ members in clade I and II contained the most exons, ranging from 10 to 15 , while $S P L$ members in the remaining clades possess 2 to 7 exons (except IbSPL19). Our results suggested that exon/intron gain or loss had occurred during the course of the Ipomoea $S P L$ gene evolution, which may result in the functional divergence of $S P L$ genes.

To investigate the features of Ipomoea SPL proteins, the conserved domains were analyzed by multiple sequence alignment. The results showed that all of the SPL members had the SBP domain, which was composed of two non-interleaved zinc finger-like structures $(\mathrm{Zn}-1 / 2)$ and one nuclear localization signal (NLS) motif (Figure 3b, Supplementary Figure S1). Based on the alignments, the Zn-2 motif is more conserved than the Zn-1 and NLS motif in all Ipomoea SPLs analyzed, which was also observed in Rosaceae [30] and Oryza species [31] (Supplementary Figure S1). For example, the Zn-2 motif in all Ipomoea SPLs was a Cys-Cys-His-Cys (C2HC) type (except IbSPL23); and the Zn-1 motif showed different signatures, which was Cys-Cys-Cys-Cys (C4) type in clade I and Cys-Cys-Cys-His (C3H) type in the remaining clades. Moreover, other conserved domains were identified in specific clades. For instance, SPLs in clade I and II possess an DEXDc domain (Supplementary Figure S2), which were involved in ATPdependent DNA unwinding [32]; SPLs in clade II contain Ankyrin repeats (Supplementary Figure S3), which were considered to be significant for mediating protein-protein interactions [33].

To gain a better understanding of Ipomoea SPL protein characteristics, the MEME software was used to explore the motif compositions (Figure 3d, Supplementary Figure S4). It was found that Ipomoea SPL proteins within the same clade showed similar motif compositions, while Ipomoea SPL proteins in different clades exhibited distinct differences in motif composition. In detail, two motifs (motif 2 and 3 ) existed in all Ipomoea SPL proteins, which were actually part of the SBP domain (Supplementary Figure S1); four motifs (motif 4, 5, 6 and 7) existed in clade I and II, and motif 4 was actually DEXDc domain (Supplementary Figure S2); motif 8 existed in clade II, which were make-up of Ankyrin repeats (Supplementary Figure S3); motif 10 presented in clade IV with unknown function. In summary, our results revealed that some motifs only presented in the specific clade, indicating that the $S P L$ genes may be significantly different in function between different clades. 


\subsection{Gene duplication, orthology relationship and selective pressure of Ipomoea SPLS}

To investigate gene duplication modes for Ipomoea SPL genes, MCScanX [34] was used to perform gene collinearity analysis within each Ipomoea species. All of the Ipomoea SPL genes were estimated to be duplicated (no singleton mode was found), and segmental $(51,48.57 \%)$ is the dominant mode compared to the other duplication modes: dispersed $(39,37.14 \%)$, tandem $(13,12.38 \%)$ and proximal $(2,1.90 \%)$ (Figure 4ab, Supplementary Table S1, Supplementary Figure S5). Our results indicated that segmental duplication has played a predominant role in the evolution and expansion of Ipomoea SPL genes. In addition, we found that tandem duplication was the most frequent event in IV-b and IV-c subclades, suggesting that $S P L$ genes in these two clades were expanded by tandem duplication.

To assign the orthology relationships of the SPL genes, OrthoMCL [35] was used to detect orthologous groups across $O$. sativa, $A$. thaliana, $S$. Iycopersicum and the four Ipomoea species. A total of $25(1,2,2,8$, 3, 5, 2 and 2) orthologous groups in the eight clades (clade I to VIII) were identified, respectively (Supplementary Table S3). Among these groups, nine groups had genes start from $O$. sativa, suggesting that $S P L$ genes in these groups may have occurred prior to the split of monocots and dicots; four groups had genes from $A$. thaliana but absent in $O$. sativa, implying that they originated after the divergence between monocots and dicots; ten groups had genes only existed in genus Ipomoea, indicating that they may originated in the common ancestor of Ipomoea lineage. Furthermore, the potential functions of some Ipomoea SPL genes could be inferred from their orthologs in $O$. sativa, $A$. thaliana and S. Iycopersicum species.

To understand the divergence of Ipomoea SPL genes, the $\mathrm{Ka}, \mathrm{Ks}$ and $\mathrm{Ka} / \mathrm{Ks}$ ratio for all orthologous groups were calculated using PAML software [36] (Figure 4c, Supplementary Table S4). As a result, the mean $\mathrm{Ka} / \mathrm{Ks}$ values for all clades were lower than 1.0, suggesting that Ipomoea SPL genes were evolved under the pressure of purifying selection. Genes in clade VIII showed the lowest mean Ka/Ks values (0.15) than those in other clades, indicating that they may evolve under the strong positive selection. In contrast, genes in subclade IV-b and IV-c exhibited the highest Ka/Ks values, implying that these two subclades have generally diverged much more rapidly than other clades.

\section{5 miR156 target site of Ipomoea SPL genes}

Using the publicly available miRNA transcriptomes (Supplementary Table S5), a total of 9 lbmiR156 members were identified in I. batatas (Figure 5a). To explore the roles of miR156-mediated posttranscriptional regulation of SPLs in the genus Ipomoea, the transcripts of all the 105 Ipomoea SPL genes were searched for the target site of miR156 using psRNATarget [37]. As a result, a total of 69 SPL genes was found to be the potential targets of miR156, including 10 InSPLs, 19 ItbSPLs, 18 ItfSPLs and 22 IbSPLS (Figure 5b, Supplementary Table S1). Among the miR156 target SPL genes, most of which (84 \%) the sites recognized by miR156 were located downstream of SBP domain in the CDS region, then followed by the 3'-UTR (Figure 5b). 
To further validate the predicted miR156-SPL interactions in I. batatas, the publicly available degradomes were used (Supplementary Table S5). The results showed that seven miR156-SPL interactions predicted by psRNATarget were confirmed by degradome sequencing (Figure 5c, Supplementary Table S1). To be more specific, the miR156 target sites of IbSPL9, IbSPL10, IbSPL12, and IbSPL15 were located in the CDS region, while the target sites of IbSPL16, IbSPL17, and IbSPL28 were located in the 3'-UTR region.

\subsection{Cis-acting elements in promoters of Ipomoea SPL genes}

To understand the regulatory mechanism and potential function of Ipomoea $S P L$ genes, cis-acting elements were analyzed in the 2000 bp upstream sequence from the start codon for all SPL genes by using PlantCARE database [38]. A total of 4088 putative cis-acting elements were identified, and then were divided into four categories: light responsiveness, plant growth, phytohormone, and abiotic/biotic stress response (Supplementary Table S6, S7). As shown in Figure 6a, SPL genes in the same clades exhibited similar cis-acting element compositions in the promoter, indicating their conserved biological functions during the evolution in the Ipomoea. Among these categories, abiotic/biotic stress response category covered the largest portion (43.66\%), followed by the light response (25.93\%), phytohormone response $(20.77 \%)$, and plant growth (9.64\%) category (Figure 6 b). In the abiotic/biotic stress response category, elements such as MYB/MYC (responds to abiotic stress signals), STRE (metal-responsive element), WUN-motif (wound-responsive element) and LTR (low-temperature-responsive element) were found. In the light responsiveness category, a series of elements were identified, such as Box 4, G-box, GT1motif, TCT-motif, GATA-motif, and MRE, of which Box 4 motif was the most common (24\%). As for the phytohormone response category, the ABRE element (responds to ABA), CGTCA-motif (responds to MeJA), ERE (responds to ethylene), TCA-element (responds to salicylic acid), and as-1 (responds to auxin) were the most common cis-acting elements, appeared in more than 60 Ipomeoa SPL genes. In the plant growth category, ARE element essential for the anaerobic induction, CAT-box related to meristem expression, and 02-site involved in zein metabolism regulation were the three major elements. In conclusion, the cisacting elements analysis suggested that Ipomoea SPL genes participated in various biological processes.

\subsection{Expression profiles of IbSPL genes in different tissues}

Among the four Ipomoea species, I. batatas is the most important cultivated crop worldwide. To explore the putative roles of the IbSPL genes, the tissue-specific expression patterns of IbSPLs were analyzed in 8 tissues (four aboveground and four underground tissues) of two sweet potato cultivars (Xuzi3 and Yan252) using publicly available transcriptomic data (Supplementary Table S8) [39]. FPKM (Fragments Per Kilobase of transcript per Million mapped reads) values were calculated and then used to evaluate gene expression levels (Supplementary Table S9). As shown in Figure 7a, the expression patterns of IbSPLs could be classified into three groups. The first group included $7 \mathrm{IbSPL}$ genes (IbSPL6/ IbSPL 15/ IbSPL22/ IbSPL23/ IbSPL24/ IbSPL25/ IbSPL26), with lowest expression levels $\left(\log _{2}(\mathrm{FPKM})<2\right)$ in all tissues. The second group was expressed at relatively high levels in all tissues, which was composed of 5 IbSPL genes (IbSPL4/ IbSPL8/IbSPL11/IbSPL17/IbSPL20). The remaining 17 
IbSPL genes belonging to the third group, which were highly expressed in some aboveground tissues, especially in shoot or young leaf. In addition, the gene expression profiles in underground tissues (fibrous and tuberous root) were investigated, and a total of $7 \mathrm{IbSPL}$ genes were found to be expressed at a high level in underground tissues (mean FPKM > 10), such as IbSPL 1, IbSPL4, IbSPL11, IbSPL 17, IbSPL20, IbSPL21 and IbSPL28, implying their potential functions in root development. Furthermore, our results showed that IbSPL genes within the same clades exhibit distinctive expression patterns, such as IbSPL14, IbSPL17, IbSPL28, and IbSPL29 in clade VI.

To further investigate the expression profiles of $I b S P L$ genes in underground tissues, publicly available transcriptomes of eight different stages during root development from cultivar "Beauregard" were used (Figure 7b, Supplementary Table S8, S9). The results showed that overall expression patterns of $I b S P L$ genes in roots of cultivar "Beauregard" were similar to that in roots of cultivar "Xuzi3" and "Yan252". To be specific, the expression levels of IbSPL17, IbSPL28, and IbSPL29 were the highest in storage root compared with undifferentiated and fibrous root; the expression level of IbSPL 1 was the highest in undifferentiated root; the expression level of $I b S P L 10$ was the highest in fibrous root; the expression levels of IbSPL4, IbSPL8, IbSPL11, IbSPL20, and IbSPL21 had no distinct variation in all tissues. Our results implied that these genes may play important roles in root development.

To confirm the expression patterns of $I b S P L s$ derived from transcriptomic data, qRT-PCR analysis was performed for 11 selected $I b S P L$ genes

(IbSPL 1, IbSPL4, IbSPL5, IbSPL 12, IbSPL 16, IbSPL17, IbSPL20, IbSPL21, IbSPL27, IbSPL28, and IbSPL29) in 11 tissues of cultivar "Xuyu34" (Figure 8). The results showed that the expression patterns of IbSPL genes were consistent between transcriptomic data and qRT-PCR results. We found the expression levels for $I b S P L s$ were quite different in various tissues. For example, IbSPL27 and IbSPL29 were highest expressed in young leaf; IbSPL12, IbSPL 16, and IbSPL21 were highest expressed in flower. Moreover, the expression levels of $I b S P L 17$ and $I b S P L 28$ were gradually increased during root development, indicating their differential roles in storage root development in sweet potato.

\subsection{IbSPL genes in response to exogenous phytohormones}

Phytohormones are known as regulators in plant growth and developmental processes, and the promoter analysis revealed that $I b S P L$ genes might be regulated by various phytohormones. To reveal the potential roles of the IbSPLs in hormonal signaling pathways, qRT-PCR was used to analyze the expression of the 6 IbSPL genes (IJSPL 1, IbSPL4, IbSPL 16, IbSPL17, IbSPL21 and IbSPL28) under exogenous phytohormone treatments, including indole-3-acetic acid (IAA), methyl-Jasmonate (MeJA), zeatin (ZT), and abscisic acid (ABA). In general, expression analysis indicated that the $6 \mathrm{IbSPL}$ genes exhibited highly divergent response patterns under the phytohormone treatment in root (Figure 9). Under IAA treatment, IbSPL 16 and IbSPL21 were rapidly upregulated after $6 \mathrm{~h}$ of IAA treatment, while the other four genes were upregulated after 12 or $24 \mathrm{~h}$ of IAA treatment. Under MeJA treatment, all IbSPL genes were significantly upregulated after $48 \mathrm{~h}$ of MeJA treatment, of which IbSPL4 and IbSPL21 were upregulated 45.3 and 178.1 folds. Under $\mathrm{ZT}$ treatment, all IDSPL genes were highly upregulated after $6 \mathrm{~h}$ of ZT treatment, then gradually declined, 
and finally were expressed at least 10 folds than CK. In particular, IbSPL21 showed extremely positive response to $Z T$ treatment. Under ABA treatment, all examined IbSPL genes, particularly IbSPL1, IbSPL4, IbSPL 16, IbSPL17, and IbSPL21, were rapidly upregulated after $6 \mathrm{~h}$ of ABA treatment, whereas IbSPL28 was significantly upregulated after $24 \mathrm{~h}$ of ABA treatment.

\subsection{Regulatory sub-networks involving IbSPLs and other I. batatas genes in the storage root of sweet potato}

To identify the regulatory sub-networks involving $I b S P L s$ in the storage root of sweet potato, weighted gene co-expression network analysis (WGCNA) was performed based on transcriptomic data of mature storage root from 88 sweet potato accessions (Supplementary Figure S6, Supplementary Table S11). A total of 19 modules were obtained, among which three modules (turquoise, blue and yellow) were found contain 8 IbSPL genes in total (Figure 10a). In the yellow module, IbSPL20 had only one co-expressed gene. In the blue module, IbSPL9 and IbSPL 10 were co-expressed with 17 and 107 genes, respectively. In the turquoise module, IbSPL 1, IbSPL 16, IbSPL 17, IbSPL21, and IbSPL28 had 12, 147, 370, 732, and 198 co-expressed genes, respectively (Supplementary Table S12). Interestingly, IbSPL 16, IbSPL 17, IbSPL21, and IbSPL28 in the turquoise module share 101 co-expressed genes (Figure 10b), indicating they may belong to the same biological process.

To further explore putative functions of the SPLs in the storage root, GO enrichment analysis was performed for the co-expressed genes. For the IbSPL 1, IbSPL20 and IbSPL9, we can't obtain G0 enrichment results due to the small number of co-expressed genes. For the IbSPL10 in the blue module, co-expressed genes related to "response to chitin" and "response to organonitrogen compound" were enriched (Supplementary Table S13). For the IbSPL16, IbSPL17, IbSPL21, and IbSPL28 in the turquoise module, the similar GO terms were enriched, such as "regulation of root morphogenesis", "cell division", "cytoskeleton organization", "plant-type cell wall organization or biogenesis", and "cellulose biosynthetic process" etc. (Figure 10c, Supplementary Table S13). It is known that the cytoskeleton, cell division, and cell wall organization/biogenesis are important biological processes in the storage root formation and development [40]. Our results indicated that IbSPL 16/IbSPL 17/IbSPL21/IbSPL28 may play a key role in storage root development in sweet potato.

\section{Discussion}

The SPL genes are important plant-specific transcription factors with highly conserved SBP domain. Since the $S P L$ genes was first discovered in A. majus, more and more members of $S P L$ gene family have been identified in plants [6-12]. However, comprehensive molecular evolutionary and function analysis of the $S P L$ genes in genus Ipomoea have not been reported. The genus Ipomoea has significant nutritional and economic value for human, including the seventh most important crop $I$. batatas and ornamental plant $I$. nil. Up to now, four species in Ipomoea have been sequenced, including I. nil, I. triloba, I. trifida and I. batatas. Utilizing these genomes, we systematically analyzed the Ipomoea $S P L$ genes, such as molecular characteristics, evolutionary process, posttranscriptional regulation and physiological function. 


\subsection{Comparative analysis of $S P L$ genes in the genus Ipomoea}

Recently, some important gene families of transcription factors have been investigated in Ipomoea species, such as bZIPs [41], WRKYs [42], GRPs [43], MADS-[44] and DEAD- box [45]. However, these studies only focus on gene family in a single Ipomoea species, the comparative analysis of gene family in genus Ipomoea are still rare. In our study, to dissect the evolutionary dynamics of the SPL genes in genus Ipomoea, SPL genes in four Ipomoea species were identified, including 29 IbSPLs, 27 ItfSPLs, 26 ItbSPLs and 23 IniSPLs (Fig. 1). It should be noted that the number of $S P L$ genes in sweet potato almost equals to that in its three diploid wild relatives (I. nil, I. triloba, and I. trifida) due to the haplotype-resolved genome assembly of hexaploid sweet potato [28]. In accordance with the classification in $A$. thaliana, $S$. lycopersicum and O. sativa [7-9], the Ipomoea SPL genes were also divided into eight clades (Fig. 2). We observed that clade IV was the largest containing 32 members, and clade I was the smallest containing only four members. By gene and protein structure analysis, it was found that most Ipomoea SPLs from the same phylogenetic clades share the similar intron/exon structures, domain organizations, and motif compositions (Fig. 3), indicating that SPLs within the same clade may have similar functions in Ipomoea species. Interestingly, except for the conserved domain and motifs presented in all SPL proteins, some other domains or motifs were found in clade I, II and IV, such as DEXDc domain (motif 4) and Ankyrin repeats (motif 8), which was also observed in papaya [46] and barley [47]. Our results suggesting that the SPLs in these clades may have undergone functional differentiation and neofunctionalization during evolution.

Ipomoea species were found possess more $S P L$ genes than that in dicotyledonous model plants, such as A. thaliana and $S$. lycopersicum [7, 9], implying genus-specific expansion of $S P L$ gene family occurred in Ipomoea. It is known that the expansion of gene family is mainly the result of duplication events during the evolution [48]. Our results showed that segmental duplication play a major role in the evolution and expansion of Ipomoea SPL genes (Fig. 4b), which was consistent with findings in cotton [49], Rosacea species [50] and Euphorbiaceae species [51]. Previous study revealed that a whole-genome triplication (WGT) event occurred 46.1 million years ago (Mya) in the progenitor of the genus Ipomoea [28], and we proposed that segmental duplication of SPLs may be derived from the WGT event. Furthermore, we found that tandem duplication was the most frequent event in IV-b and IV-c subclades (Supplementary Table S1), and no orthologs were found in dicots (Supplementary Table S3), implying that SPL members in these two subclades were tandem duplicated from a recent event. We speculated that $S P L$ genes undergone replication expansion in the progenitor of the genus Ipomoea, and different SPL members were retained due to their important role in growth and development during the Ipomoea species differentiation. In addition, the Ka/Ks ratios were less than 1 for all Ipomoea SPL ortholog gene pairs, indicating that Ipomoea SPL genes were under strong purifying selection (Supplementary Table S4).

Many $S P L$ genes are targeted by miR156, thus forming a functional regulatory network of miR156-SPL, which play an important role in plant growth and development $[2,21,22]$. It has been reported that more than half of $S P L$ gene family members was targeted by miR156 in various plant species, such as rice [8], 
tomato [9], and apple [10]. In this study, two-thirds of $S P L$ genes in each Ipomoea species were predicted as the targets of miR156 (Fig. 5b). Phylogenetic analysis showed that all the members of $S P L$ genes in clades III lacking miR156 binding sites, consistent with the results from $A$. thaliana, rice, and tomato [4]. In addition, two kinds of binding site of miR156 were identified in SPL genes, one locates in CDS and the other locates in 3'-UTR (Fig. 5b), which was also observed in other plants, such as rice [8], tomato [9], apple [10] and papaya [46]. With the degradome data in I. batatas, $7 \mathrm{IbSPL}$ genes were further confirmed as the targets of miR156 (Fig. 5c, Supplementary Table S1). Among these IbSPL genes, the miR156 binding site for IbSPL17 and IbSPL28 was located in 3'-UTR, and the miR156 binding site of their orthologous genes (AtSPL3 and LeSPL-CNR) in A. thaliana and tomato are also located in 3'-UTR [5, 52], suggesting the high conservation of miR156-SPL regulatory module in plants. However, most of the miR156-SPL interactions in this study were predicted by in silico analysis, more experimental verification needs to be performed for the miR156-SPL interactions in genus Ipomoea.

\subsection{IbSPL genes associated with storage root development}

Sweet potato, is the seventh most important crop globally, has a strong adaptability, stable yield, and high nutritional value [25]. The storage root of sweet potato is the most important economic trait in nutrient content and yield, and dissecting the mechanisms underlying the formation and development of storage roots is of great significance to improve the nutrient content and yield of sweet potato. Considering the key regulatory roles of $S P L$ genes in root architecture $[13,14]$ and biomass enhancement [20], the expression patterns of IbSPL genes at different tissues or developmental stages were evaluated. Using the public transcriptome data, we found that most of the IbSPLs were highly expressed in aboveground tissues, especially in shoot or young leaf; however, only some IbSPLs were found to be expressed at a high level in underground tissues (Fig. 7). With a qRT-PCR assay, the expression levels of three IbSPL genes (IbSPL17/IbSPL28) were obviously increased with storage development (Fig. 8). The results from our study provide evidence that $S P L$ genes may have important functions during the storage root development in sweet potato.

The formation and development of storage roots is a complex physiological process that includes cessation of root elongation, genesis and development of the primary and secondary vascular cambium, increase in radial growth, and accumulation of starch and storage proteins [53]. It is well known that these processes are closely related to the endogenous phytohormones, such as IAA, cytokinins (CTKs), JA, and $A B A[40,53-55]$. For instance, IAA involved in the early stage of storage root formation and the primary thickening of storage roots [56], ABA had a significant role in in storage root bulking by activating cell division [57], and CTKs played a key role in the initiation and expansion rate of storage roots as a prerequirement for cambial cell proliferation [40]. It is also reported that storage root yields are positively correlated with the contents of ABA and CTKs [58]. In the present study, different kinds of hormoneresponsive elements were found by analyzing the $I b S P L$ promoters (Fig. 6), implying $I b S P L$ genes may participate in hormonal signaling pathways. qRT-PCR further confirmed that the expression of all tested IbSPLS (IbSPL 1, IbSPL4, IbSPL 16, IbSPL17, IbSPL21 and IbSPL28) was strongly induced under exogenous phytohormone treatments, especially $Z \mathrm{ZT}$ and $\mathrm{ABA}$, suggesting their crucial roles in root development. 
The formation and development of storage roots are maintained by coordinated cellular behaviors, such as cell division, expansion, and differentiation. Previous studies revealed that cell wall biosynthesis and cytoskeleton organization are critical in these cellular behaviors $[59,60]$. In this study, regulatory subnetworks analyzed by WGCNA indicated that $8 \mathrm{IbSPL}$ genes were co-expressed with at least one other $I$. batatas genes (Fig. 10a). GO enrichment analysis of co-expressed genes revealed that these IbSPL genes may be involved in stress responses, root morphogenesis, and cell division (Supplementary Table S13). Moreover, genes co-expressed with IbSPL16/ IbSPL17/IbSPL21/IbSPL28 in the turquoise module were all significantly enriched for "regulation of root morphogenesis", "cell division", "cytoskeleton organization", "plant-type cell wall organization or biogenesis", and "cellulose biosynthetic process". These enriched processes are essential for cell morphogenesis and cell cycles, implying their key roles in storage root development. By combining the results from expression patterns under different root developmental stages or various phytohormones treatments and regulatory sub-networks, we concluded that IbSPL 16/ IbSPL 17/ IbSPL21/IbSPL28 may play important role in the formation and development of storage roots. In the future, functional characterization is needed to elucidate the specific roles of IbSPLs in storage root development.

\section{Conclusions}

In summary, we performed a genome-wide analysis of $S P L$ gene family in four Ipomoea species, including I. batatas, I. trifida, I. triloba, and I. nil. A total of 105 Ipomoea SPL genes were identified, and could be divided into eight clades. Genes in one clade were found harbor similar gene structures, domain organizations, motif compositions and cis-acting elements, suggesting similarity of biological function potentially. Segmental duplication was the major force accounting for the expansion of Ipomoea SPL gene family. By combining the results from expression patterns and regulatory sub-networks, we concluded that IbSPL 16/ IbSPL17/ IbSPL21/ IbSPL28 may play important role in the formation and development of storage roots. Taken together, our results not only provide novel insights into the evolutionary and functional divergence of the $S P L$ genes in genus Ipomoea, but also lay a foundation for further elucidation of the potential functional roles of $I b S P L$ genes during storage root development.

\section{Materials And Methods}

\subsection{Identification of SPL genes in I. nil, I. triloba, I. trifida, and I. batatas}

The genomes of four Ipomoea species (including I. nil, I. triloba, I. trifida and I. batatas) were downloaded from "Ipomoea nil Genome Project" (http://viewer.shigen.info/asagao) [27], "Sweetpotato Genomic Resource" (http://sweetpotato.plantbiology.msu.edu) [29] and "Ipomoea Genome Hub" (http://ipomoeagenome.org) [28], respectively. To identify the $S P L$ genes in above four species, three methods described below were used. First, all of the 16 A. thaliana SPL proteins (https://www.arabidopsis.org/) were used as queries to find $S P L$ homologs using BLASTP program with a threshold of e-value $<1 \mathrm{e}-3$. Second, the Hidden Markov Model (HMM) of SBP (PF03110) domain was downloaded from Pfam database [61] and was used to identify putative SPL proteins by hmmsearch [61] program. Finally, all of the candidate SPL 
proteins obtained from BLASTP and hmmsearch were submitted to SMART [62] and ScanProsite database [63] to confirm the presence of SBP domain. Those proteins lacking SBP domain were excluded, and the remaining were considered as the SPL proteins. In addition, the structures of identified IbSPLs were further examined manually to correct genome assembly errors.

The BUSCA online software [64] was used to predict subcellular localization of Ipomoea SPL proteins. An in-house Perl script was used to analysis the physical and chemical properties of Ipomoea SPL proteins, such as protein length, molecular weight (MW, $\mathrm{kD})$ and isoelectric point (pl).

\subsection{Phylogenetic analysis of SPL proteins}

For the SPL proteins from C. reinhardtii, A. thaliana, S. Iycopersicum, O. sativa, and the four Ipomoea species, phylogenetic analysis was performed using the following steps: first, the mafft software (v7.45) [65] was used to perform the multiple alignment for full-length SPL proteins; second, the Gblocks program (v0.71b) [66] was used to select conserved blocks from the multiple alignment; third, MEGA X software [67] was used to construct a neighbor-joining phylogenetic tree with 1000 bootstrap replications, CRR1 protein from $C$. reinhardtii was set as an outgroup; finally, the tree was visualized using Evolview website [68]. For the Ipomoea SPL proteins, a neighbor-joining phylogenetic tree was constructed using the methods as described above.

\subsection{Analysis of the gene structure, protein domain and motif}

The exon/intron positions of all Ipomoea SPL genes were obtained based on the GFF3 files downloaded from the genomic database. The domain organizations of Ipomoea SPL proteins were annotated based on the results from SMART database [62]. The conserved sequences in each domain were shaded at four levels by GeneDoc. The motif compositions of Ipomoea SPL proteins were analyzed by MEME online database [69], with the maximum number set to 10. Finally, the gene structure, domain organization, and motif composition were drawn using Tbtools [70].

\subsection{Gene duplication, orthology and selection analysis}

The MCScanX software [34] was used to identify collinear blocks within or between species, and then classify the $S P L$ genes into five different types: singleton, dispersed, proximal, tandem and segmental duplication. The synteny relationships of the collinearity blocks in each Ipomoea species were visualized using Circos [71]. The OrthoMCL software [35] was used to detect orthologous groups between diverse SPL genes. For each orthologue gene pairs, the Ks (synonymous substitution rate), Ka (nonsynonymous substitution rate) and $\mathrm{Ka} / \mathrm{Ks}$ ratio (evolutionary constraint) were calculated by PAML [36].

\subsection{Prediction of miR156-targeted genes}


The publicly available datasets were used to identify miR156 sequence in Ipomoea. First, a total of 58 miRNA transcriptomes deposited in NCBI [72] (16 in PRJNA471495, 2 in PRJNA474012, 11 in PRJNA592001, 12 in PRJNA599544, 12 in PRJNA600587, 5 in PRJNA638516) were collected from previous studies (Supplementary Table S5) [73-76]. Then, Trimmomatic (version 0.39) [77] was used to filter the raw miRNA sequencing data to remove low-quality reads and sequencing adaptors. Finally, using I. batatas as the reference genome, miRDeep2 (version 1.1.4) pipeline [78] was employed to identify miR156 sequence with default parameters.

For the Ipomoea SPL genes, the miR156 target sites were predicted using the psRNATarget server [37] with default settings. To validate the predicted miR156-SPL interactions in I. batatas, a total of 5 degradomes (1 in PRJNA592001, 4 in PRJNA600587) $[74,76]$ were downloaded from the public database were used (Supplementary Table S5). After filtering out the low-quality reads and sequencing adaptors, CleaveLand4 pipeline [79] was used to identify miR156 cleavage sites. The identified targets with category $0-3$ and $p$-value $<0.05$ were considered as the reliable miR156 target genes.

\subsection{Promoter analysis of Ipomoea SPL genes}

The 2000 bp upstream sequence from the start codon for all Ipomoea SPL genes were retrieved from the genome using an in-house Perl script, and then were submitted to PlantCARE program [38] for predicting cis-acting elements as previously described [80]. The distribution of cis-acting elements in each promoter was drawn using TBtools [70].

\subsection{Plant materials and hormone treatments}

Sweet potato (I. batatas cv. Xuyu34) plants used in this study were provided by the Xuzhou Academy of Agricultural Sciences, Xuzhou, Jiangsu, China. According to institutional, national and international guidelines, these samples do not require specific permissions for research purposes. The plants were grown in greenhouses on the campus of Jiangsu Normal University, Xuzhou, China. For organ-specific expression analysis, the tissues of young leaves, mature leaves, flowers, roots (10DAT, 20DAT, 40DAT, 60DAT, 80DAT, 90DAT and 100DAT roots with $0.3 \mathrm{~mm}, 2 \mathrm{~mm}, 7 \mathrm{~mm}, 25 \mathrm{~mm}, 37 \mathrm{~mm}, 52 \mathrm{~mm}$ and $60 \mathrm{~mm}$ in diameter, respectively) were collected. For hormone treatments, adventitious roots were first induced from stem cuttings grown hydroponically for ten days, and then were treated with $100 \mu \mathrm{M}$ ABA, IAA, ZT, and MeJA, respectively. Adventitious roots were collected at $0,6,12,24$, and $48 \mathrm{~h}$ post the treatment. Three biological replicates were collected for each sample. All samples were frozen in liquid nitrogen and finally stored at $-80^{\circ} \mathrm{C}$ for subsequent use.

\subsection{RNA extraction and qRT-PCR analysis}

Total RNA for each sample was extracted using RNApure Plant Kit (CWBio, Beijing, China), according to the manufacturers' instructions. The first strand cDNA was synthesized from $1.0 \mu \mathrm{g}$ total pure RNA using the 
HiFiScript cDNA Synthesis Kit (CWBio, Beijing, China). Gene-specific primers for each IbSPL gene were designed using primer3 [81]. The qRT-PCR was performed on Bio-rad CFX Connect ${ }^{\text {TM }}$ Real-Time System (Bio-Rad, CA, USA) using $2 \times$ Q3 SYBR qPCR Master Mix (Universal) (Tolo Biotechnology, Shanghai, China) premix. The IbARF gene was used as a reference gene for normalizing the expression levels [82]. The relative transcript abundance for each $I b S P L$ gene was calculated using the $2^{-\triangle \triangle C T}$ approach [83]. Statistical analysis was conducted by ANOVA followed by LSD test, and different letters indicate statistically significant difference at $p<0.05$. The used primers were listed in Supplementary Table S10.

\subsection{Analysis of expression patterns of IbSPLs using published transcriptomic data}

To explore tissue- and developmental stage-specific expression patterns of IbSPL genes, publicly available transcriptome datasets from two previous studies [29, 39] (Supplementary Table S8) were used: one was 8 different tissues from cultivar Xuzi3 and Yan252 under BioProject accession number PRJCA000640 in NGDC [84], and the other was 8 different stages during root development from cultivar Beauregard under BioProject accession number PRJNA491292 in NCBI [72]. Transcriptome analysis was performed according to our previous study [26]. The downloaded raw fastq files were filtered using Trimmomatic (version 0.39) [77], and then were mapped to sweet potato genome Taizhong6 [28] using STAR (version 2.7.1a) software under the 2-pass mapping mode [85]. RSEM [86] was used to calculate FPKM values for each gene. Finally, heatmap was plotted based on the normalized expression values of $29 \mathrm{IbSPL}$ genes using pheatmap package in $\mathrm{R}$.

\subsection{Construction of co-expression networks involving IbSPL and other $I$. batatas genes in the storage root of sweet potato}

Transcriptomic dataset of mature storage roots of 88 sweet potato accessions were obtained from a previous study (Supplementary Table S11) [39] under BioProject accession number PRJCA000642 in NGDC [84]. Weighted co-expression network construction and module detection were performed using the R package WGCNA (version 1.4.9) [87] with the following parameters: the power was set as 9 , the minModuleSize was set as 30 , the cutHeight was set as 0.25 , and the network module export weight threshold was set as 0.05 . The sub-network was subsequently visualized using Cytoscape [88]. eggNOGmapper (version 2) [89] was used to assign the functional annotation of all sweet potato genes, and Clusterprofiler [90] was used to perform GO enrichment analysis for genes co-expressed with IbSPLS with adjusted $P$-value $<0.05$.

\section{List Of Abbreviations}




\begin{tabular}{l|l}
\hline Abbreviation & Explanation \\
\hline SPL & SQUAMOSA promoter-binding protein-like \\
\hline MW & Molecular Weight \\
\hline pI & The isoelectric point \\
\hline FPKM & Fragments Per Kilobase of transcript per Million mapped reads \\
\hline IAA & Indole-3-cetic Acid \\
\hline MeJA & Methyl-Jasmonate \\
\hline ABA & Abscisic Acid \\
\hline ZT & Zeatin \\
\hline WGCNA & Weighted Gene Co-expression Network Analysis \\
\hline
\end{tabular}

\section{Declarations}

\section{Ethics approval and consent to participate}

Not applicable.

\section{Consent for publication}

Not applicable.

\section{Availability of data and materials}

The datasets generated or analysed during this study are included in this published article (and its supplementary information files), or are available from the corresponding author on reasonable request.

\section{Competing interests}

The authors declare that they have no competing interests.

\section{Funding}


This work was supported by the Natural Science Foundation of Jiangsu Province (No. BK20190995), the Shanghai Municipal Afforestation \& City Appearance and Environmental Sanitation Administration (No. G212402), the Natural Science Foundation of Xuzhou City (No. KC19070), Postgraduate Research \& Practice Innovation Program of Jiangsu Province (No. KYCX20_2319), and the Priority Academic Program Development of Jiangsu Higher Education Institutions (PAPD). All the funders had no role in the design of the study and collection, analysis, and interpretation of data and in writing the manuscript.

\section{Authors' contributions}

L.Z., S.W., and T.X. conceived and designed the research. H.S., J.M., L.Z., W.H., and Y.Z. performed the research and analyzed the data. L.Z. and H.S. wrote the manuscript. All authors read and approved the manuscript.

\section{Acknowledgements}

We would like to thank Dr. Meng Kou (Xuzhou academy of agricultural sciences) for providing the sweet potato (I. batatas cv. Xuyu34) plants.

\section{References}

1. Guo AY, Zhu QH, Gu X, Ge S, Yang J, Luo J: Genome-wide identification and evolutionary analysis of the plant specific SBP-box transcription factor family. Gene 2008, 418(1-2):1-8.

2. Wang H, Wang H: The miR156/SPL Module, a Regulatory Hub and Versatile Toolbox, Gears up Crops for Enhanced Agronomic Traits. Molecular plant 2015, 8(5):677-688.

3. Chen X, Zhang Z, Liu D, Zhang K, Li A, Mao L: SQUAMOSA promoter-binding protein-like transcription factors: star players for plant growth and development. Journal of integrative plant biology 2010, 52(11):946-951.

4. Preston JC, Hileman LC: Functional Evolution in the Plant SQUAMOSA-PROMOTER BINDING PROTEIN-LIKE (SPL) Gene Family. Frontiers in plant science 2013, 4:80.

5. Chen W, Kong J, Lai T, Manning K, Wu C, Wang Y, Qin C, Li B, Yu Z, Zhang X et al: Tuning LeSPL-CNR expression by SlymiR157 affects tomato fruit ripening. Scientific reports 2015, 5:7852.

6. Klein J, Saedler H, Huijser P: A new family of DNA binding proteins includes putative transcriptional regulators of the Antirrhinum majus floral meristem identity gene SQUAMOSA. Mol Gen Genet 1996, 250(1):7-16.

7. Cardon G, Hohmann S, Klein J, Nettesheim K, Saedler H, Huijser P: Molecular characterisation of the Arabidopsis SBP-box genes. Gene 1999, 237(1):91-104. 
8. Xie K, Wu C, Xiong L: Genomic organization, differential expression, and interaction of SQUAMOSA promoter-binding-like transcription factors and microRNA156 in rice. Plant physiology 2006, 142(1):280-293.

9. Salinas M, Xing S, Hohmann S, Berndtgen R, Huijser P: Genomic organization, phylogenetic comparison and differential expression of the SBP-box family of transcription factors in tomato. Planta 2012, 235(6):1171-1184.

10. Li J, Hou H, Li X, Xiang J, Yin X, Gao H, Zheng Y, Bassett CL, Wang X: Genome-wide identification and analysis of the SBP-box family genes in apple (Malus $\mathrm{x}$ domestica Borkh.). Plant physiology and biochemistry: PPB / Societe francaise de physiologie vegetale 2013, 70:100-114.

11. Zhang HX, Jin JH, He YM, Lu BY, Li DW, Chai WG, Khan A, Gong ZH: Genome-Wide Identification and Analysis of the SBP-Box Family Genes under Phytophthora capsici Stress in Pepper (Capsicum annuum L.). Frontiers in plant science 2016, 7:504.

12. Tripathi RK, Goel R, Kumari S, Dahuja A: Genomic organization, phylogenetic comparison, and expression profiles of the SPL family genes and their regulation in soybean. Dev Genes Evo/2017, 227(2):101-119.

13. Yu N, Niu QW, Ng KH, Chua NH: The role of miR156/SPLs modules in Arabidopsis lateral root development. The Plant journal: for cell and molecular biology 2015, 83(4):673-685.

14. Barrera-Rojas $\mathrm{CH}$, Rocha GHB, Polverari L, Pinheiro Brito DA, Batista DS, Notini MM, da Cruz ACF, Morea EGO, Sabatini S, Otoni WC et al: miR156-targeted SPL10 controls Arabidopsis root meristem activity and root-derived de novo shoot regeneration via cytokinin responses. J Exp Bot 2020, 71(3):934-950.

15. Shao Y, Zhou HZ, Wu Y, Zhang H, Lin J, Jiang X, He Q, Zhu J, Li Y, Yu H et al: OsSPL3, an SBP-Domain Protein, Regulates Crown Root Development in Rice. The Plant cell 2019, 31(6):1257-1275.

16. Xu X, Li X, Hu X, Wu T, Wang Y, Xu X, Zhang X, Han Z: High miR156 Expression Is Required for AuxinInduced Adventitious Root Formation via MXSPL26 Independent of PINs and ARFs in Malus xiaojinensis. Frontiers in plant science 2017, 8:1059.

17. Yamaguchi A, Wu MF, Yang L, Wu G, Poethig RS, Wagner D: The microRNA-regulated SBP-Box transcription factor SPL3 is a direct upstream activator of LEAFY, FRUITFULL, and APETALA1. Developmental cell 2009, 17(2):268-278.

18. Manning K, Tor M, Poole M, Hong Y, Thompson AJ, King GJ, Giovannoni JJ, Seymour GB: A naturally occurring epigenetic mutation in a gene encoding an SBP-box transcription factor inhibits tomato fruit ripening. Nature genetics 2006, 38(8):948-952.

19. Orfila C, Huisman MM, Willats WG, van Alebeek GJ, Schols HA, Seymour GB, Knox JP: Altered cell wall disassembly during ripening of $\mathrm{Cnr}$ tomato fruit: implications for cell adhesion and fruit softening. Planta 2002, 215(3):440-447.

20. Wang S, Li S, Liu Q, Wu K, Zhang J, Wang S, Wang Y, Chen X, Zhang Y, Gao C et al: The OsSPL16-GW7 regulatory module determines grain shape and simultaneously improves rice yield and grain quality. Nature genetics 2015, 47(8):949-954. 
21. Gou JY, Felippes FF, Liu CJ, Weigel D, Wang JW: Negative regulation of anthocyanin biosynthesis in Arabidopsis by a miR156-targeted SPL transcription factor. The Plant cell 2011, 23(4):1512-1522.

22. Liu M, Shi Z, Zhang X, Wang M, Zhang L, Zheng K, Liu J, Hu X, Di C, Qian Q et al: Inducible overexpression of Ideal Plant Architecture1 improves both yield and disease resistance in rice. Nature plants 2019, 5(4):389-400.

23. Austin DF, Huáman Z: A synopsis of Ipomoea (Convolvulaceae) in the Americas. Taxon 1996, 45(1):3-38.

24. Morita Y, Hoshino A: Recent advances in flower color variation and patterning of Japanese morning glory and petunia. Breed Sci 2018, 68(1):128-138.

25. Liu Q: Improvement for agronomically important traits by gene engineering in sweetpotato. Breed Sci 2017, 67(1):15-26.

26. Zhang L, Yu Y, Shi T, Kou M, Sun J, Xu T, Li Q, Wu S, Cao Q, Hou W et al: Genome-wide analysis of expression quantitative trait loci (eQTLs) reveals the regulatory architecture of gene expression variation in the storage roots of sweet potato. Horticulture research 2020, 7(1):90.

27. Hoshino A, Jayakumar V, Nitasaka E, Toyoda A, Noguchi H, Itoh T, Shin IT, Minakuchi Y, Koda Y, Nagano AJ et al: Genome sequence and analysis of the Japanese morning glory Ipomoea nil. Nature communications 2016, 7:13295.

28. Yang J, Moeinzadeh MH, Kuhl H, Helmuth J, Xiao P, Haas S, Liu G, Zheng J, Sun Z, Fan W et al: Haplotype-resolved sweet potato genome traces back its hexaploidization history. Nature plants 2017, 3(9):696-703.

29. Wu S, Lau KH, Cao Q, Hamilton JP, Sun H, Zhou C, Eserman L, Gemenet DC, Olukolu BA, Wang H et al: Genome sequences of two diploid wild relatives of cultivated sweetpotato reveal targets for genetic improvement. Nat Commun 2018, 9(1):4580.

30. Jiang X, Chen P, Zhang X, Liu Q, Li H: Comparative analysis of the SPL gene family in five Rosaceae species: Fragaria vesca, Malus domestica, Prunus persica, Rubus occidentalis, and Pyrus pyrifolia. Open Life Sci 2021, 16(1):160-171.

31. Zhong H, Kong W, Gong Z, Fang X, Deng X, Liu C, Li Y: Evolutionary Analyses Reveal Diverged Patterns of SQUAMOSA Promoter Binding Protein-Like (SPL) Gene Family in Oryza Genus. Frontiers in plant science $2019,10: 565$.

32. Caruthers JM, McKay DB: Helicase structure and mechanism. Curr Opin Struct Biol 2002, 12(1):123133.

33. Li J, Mahajan A, Tsai MD: Ankyrin repeat: a unique motif mediating protein-protein interactions. Biochemistry 2006, 45(51):15168-15178.

34. Wang Y, Tang H, Debarry JD, Tan X, Li J, Wang X, Lee TH, Jin H, Marler B, Guo H et al: MCScanX: a toolkit for detection and evolutionary analysis of gene synteny and collinearity. Nucleic acids research 2012, 40(7):e49.

35. Li L, Stoeckert CJ, Jr., Roos DS: OrthoMCL: identification of ortholog groups for eukaryotic genomes. Genome research 2003, 13(9):2178-2189. 
36. Yang Z: PAML 4: phylogenetic analysis by maximum likelihood. Molecular biology and evolution 2007, 24(8):1586-1591.

37. Dai X, Zhuang Z, Zhao PX: psRNATarget: a plant small RNA target analysis server (2017 release). Nucleic acids research 2018, 46(W1):W49-W54.

38. Lescot M, Dehais P, Thijs G, Marchal K, Moreau Y, Van de Peer Y, Rouze P, Rombauts S: PlantCARE, a database of plant cis-acting regulatory elements and a portal to tools for in silico analysis of promoter sequences. Nucleic acids research 2002, 30(1):325-327.

39. Ding N, Wang A, Zhang X, Wu Y, Wang R, Cui H, Huang R, Luo Y: Identification and analysis of glutathione S-transferase gene family in sweet potato reveal divergent GST-mediated networks in aboveground and underground tissues in response to abiotic stresses. BMC plant biology 2017, 17(1):225.

40. Dong T, Zhu M, Yu J, Han R, Tang C, Xu T, Liu J, Li Z: RNA-Seq and iTRAQ reveal multiple pathways involved in storage root formation and development in sweet potato (Ipomoea batatas L.). BMC plant biology 2019, 19(1):136.

41. Yang Z, Sun J, Chen Y, Zhu P, Zhang L, Wu S, Ma D, Cao Q, Li Z, Xu T: Genome-wide identification, structural and gene expression analysis of the bZIP transcription factor family in sweet potato wild relative Ipomoea trifida. BMC genetics 2019, 20(1):41.

42. Li Y, Zhang L, Zhu P, Cao Q, Sun J, Li Z, Xu T: Genome-wide identification, characterisation and functional evaluation of WRKY genes in the sweet potato wild ancestor Ipomoea trifida (H.B.K.) G. Don. under abiotic stresses. BMC genetics 2019, 20(1):90.

43. Lu Y, Sun J, Yang Z, Zhao C, Zhu M, Ma D, Dong T, Zhou Z, Liu M, Yang D et al: Genome-wide identification and expression analysis of glycine-rich RNA-binding protein family in sweet potato wild relative Ipomoea trifida. Gene 2019, 686:177-186.

44. Zhu P, Dong $T, X u T$, Kang H: Identification, characterisation and expression analysis of MADS-box genes in sweetpotato wild relative Ipomoea trifida. Acta Physiologiae Plantarum 2020, 42(11):163.

45. Wan R, Liu J, Yang Z, Zhu P, Cao Q, Xu T: Genome-wide identification, characterisation and expression profile analysis of DEAD-box family genes in sweet potato wild ancestor Ipomoea trifida under abiotic stresses. Genes Genomics 2020, 42(3):325-335.

46. $X u Y, X u H$, Wall MM, Yang J: Roles of transcription factor SQUAMOSA promoter binding protein-like gene family in papaya (Carica papaya) development and ripening. Genomics 2020, 112(4):27342747.

47. Tripathi RK, Bregitzer P, Singh J: Genome-wide analysis of the SPL/miR156 module and its interaction with the AP2/miR172 unit in barley. Scientific reports 2018, 8(1):7085.

48. Moore RC, Purugganan MD: The evolutionary dynamics of plant duplicate genes. Current opinion in plant biology 2005, 8(2):122-128.

49. Cai C, Guo W, Zhang B: Genome-wide identification and characterization of SPL transcription factor family and their evolution and expression profiling analysis in cotton. Scientific reports 2018, 8(1):762. 
50. Abdullah M, Cao Y, Cheng X, Shakoor A, Su X, Gao J, Cai Y: Genome-Wide Analysis Characterization and Evolution of SBP Genes in Fragaria vesca, Pyrus bretschneideri, Prunus persica and Prunus mume. Frontiers in genetics 2018, 9:64.

51. Li J, Gao X, Sang S, Liu C: Genome-wide identification, phylogeny, and expression analysis of the SBPbox gene family in Euphorbiaceae. BMC genomics 2019, 20(Suppl 9):912.

52. Gandikota M, Birkenbihl RP, Hohmann S, Cardon GH, Saedler H, Huijser P: The miRNA156/157 recognition element in the 3' UTR of the Arabidopsis SBP box gene SPL3 prevents early flowering by translational inhibition in seedlings. The Plant journal: for cell and molecular biology 2007, 49(4):683-693.

53. Ravi V, Naskar SK, Makeshkumar T, Babu B, Krishnan BP: Molecular physiology of storage root formation and development in sweet potato (Ipomoea batatas (L.) Lam.). 2009.

54. Tanaka M, Kato N, Nakayama H, Nakatani M, Takahata Y: Expression of class I knotted1-like homeobox genes in the storage roots of sweetpotato (Ipomoea batatas). Journal of plant physiology 2008, 165(16):1726-1735.

55. Nakatani M: Changes in endogenous level of zeatin riboside, abscisic acid and indole acetic acid during formation and thickening of tuberous root in sweet potato. Jpnjcrop 1991, 60.

56. Noh SA, Lee HS, Huh EJ, Huh GH, Paek KH, Shin JS, Bae JM: SRD1 is involved in the auxin-mediated initial thickening growth of storage root by enhancing proliferation of metaxylem and cambium cells in sweetpotato (Ipomoea batatas). J Exp Bot 2010, 61(5):1337-1349.

57. Huan L, Jin-Qiang W, Qing L: Photosynthesis product allocation and yield in sweet potato with spraying exogenous hormones under drought stress. Journal of Plant Physiology 2020, 253:153265.

58. Wang QM, Zhang LM, Wang ZL: Formation and Thickening of Tuberous Roots in Relation to the Endogenous Hormone Concentrations in Sweetpotato. Scientia Agricultura Sinica 2005.

59. Brasil JN, Costa CNM, Cabral LM, Ferreira PCG, Hemerly AS: The plant cell cycle: Pre-Replication complex formation and controls. Genet Mol Biol 2017, 40(1 suppl 1):276-291.

60. Bashline L, Lei L, Li S, Gu Y: Cell wall, cytoskeleton, and cell expansion in higher plants. Molecular plant 2014, 7(4):586-600.

61. El-Gebali S, Mistry J, Bateman A, Eddy SR, Luciani A, Potter SC, Qureshi M, Richardson LJ, Salazar GA, Smart A et al: The Pfam protein families database in 2019. Nucleic acids research 2019, 47(D1):D427D432.

62. Letunic I, Bork P: 20 years of the SMART protein domain annotation resource. Nucleic acids research 2018, 46(D1):D493-D496.

63. de Castro E, Sigrist CJ, Gattiker A, Bulliard V, Langendijk-Genevaux PS, Gasteiger E, Bairoch A, Hulo N: ScanProsite: detection of PROSITE signature matches and ProRule-associated functional and structural residues in proteins. Nucleic acids research 2006, 34(Web Server issue):W362-365.

64. Savojardo C, Martelli PL, Fariselli P, Profiti G, Casadio R: BUSCA: an integrative web server to predict subcellular localization of proteins. Nucleic acids research 2018, 46(W1):W459-W466. 
65. Katoh K, Standley DM: MAFFT multiple sequence alignment software version 7: improvements in performance and usability. Molecular biology and evolution 2013, 30(4):772-780.

66. Castresana J: Selection of conserved blocks from multiple alignments for their use in phylogenetic analysis. Molecular biology and evolution 2000, 17(4):540-552.

67. Kumar S, Stecher G, Li M, Knyaz C, Tamura K: MEGA X: Molecular Evolutionary Genetics Analysis across Computing Platforms. Molecular biology and evolution 2018, 35(6):1547-1549.

68. Subramanian B, Gao S, Lercher MJ, Hu S, Chen WH: Evolview v3: a webserver for visualization, annotation, and management of phylogenetic trees. Nucleic acids research 2019, 47(W1):W270-W275.

69. Bailey TL, Boden M, Buske FA, Frith M, Grant CE, Clementi L, Ren J, Li WW, Noble WS: MEME SUITE: tools for motif discovery and searching. Nucleic acids research 2009, 37(Web Server issue):W202-208.

70. Chen C, Chen H, Zhang Y, Thomas HR, Frank MH, He Y, Xia R: TBtools: An Integrative Toolkit Developed for Interactive Analyses of Big Biological Data. Molecular plant 2020, 13(8):1194-1202.

71. Krzywinski M, Schein J, Birol I, Connors J, Gascoyne R, Horsman D, Jones SJ, Marra MA: Circos: an information aesthetic for comparative genomics. Genome research 2009, 19(9):1639-1645.

72. Coordinators NR: Database resources of the National Center for Biotechnology Information. Nucleic acids research 2018, 46(D1):D8-D13.

73. Saminathan T, Alvarado A, Lopez C, Shinde S, Gajanayake B, Abburi VL, Vajja VG, Jagadeeswaran G, Raja Reddy K, Nimmakayala $\mathrm{P}$ et al: Elevated carbon dioxide and drought modulate physiology and storage-root development in sweet potato by regulating microRNAs. Functional \& integrative genomics 2019, 19(1):171-190.

74. Yang Z, Zhu P, Kang H, Liu L, Cao Q, Sun J, Dong T, Zhu M, Li Z, Xu T: High-throughput deep sequencing reveals the important role that microRNAs play in the salt response in sweet potato (Ipomoea batatas L.). BMC genomics 2020, 21(1):164.

75. Kuo Y-W, Lin J-S, Li Y-C, Jhu M-Y, King Y-C, Jeng S-T: MicroR408 regulates defense response upon wounding in sweet potato. Journal of experimental botany 2019, 70(2):469-483.

76. Liu Y, Su W, Wang L, Lei J, Chai S, Zhang W, Yang X: Integrated transcriptome, small RNA and degradome sequencing approaches proffer insights into chlorogenic acid biosynthesis in leafy sweet potato. PloS one 2021, 16(1):e0245266.

77. Bolger AM, Lohse M, Usadel B: Trimmomatic: a flexible trimmer for Illumina sequence data. Bioinformatics 2014, 30(15):2114-2120.

78. Kuang Z, Wang Y, Li L, Yang X: miRDeep-P2: accurate and fast analysis of the microRNA transcriptome in plants. Bioinformatics 2019, 35(14):2521-2522.

79. Addo-Quaye C, Miller W, Axtell MJ: CleaveLand: a pipeline for using degradome data to find cleaved small RNA targets. Bioinformatics 2009, 25(1):130-131.

80. Chen Y, Zhu P, Wu S, Lu Y, Sun J, Cao Q, Li Z, Xu T: Identification and expression analysis of GRAS transcription factors in the wild relative of sweet potato Ipomoea trifida. BMC genomics 2019, 20(1):911. 
81. Untergasser A, Cutcutache I, Koressaar T, Ye J, Faircloth BC, Remm M, Rozen SG: Primer3-new capabilities and interfaces. Nucleic acids research 2012, 40(15):e115-e115.

82. Park SC, Kim YH, Ji CY, Park S, Jeong JC, Lee HS, Kwak SS: Stable internal reference genes for the normalization of real-time PCR in different sweetpotato cultivars subjected to abiotic stress conditions. PloS one 2012, 7(12):e51502.

83. Livak KJ, Schmittgen TD: Analysis of relative gene expression data using real-time quantitative PCR and the $2^{-\Delta \Delta C T}$ Method. Methods 2001, 25(4):402-408.

84. National Genomics Data Center M, Partners: Database Resources of the National Genomics Data Center in 2020. Nucleic acids research 2020, 48(D1):D24-D33.

85. Dobin A, Davis CA, Schlesinger F, Drenkow J, Zaleski C, Jha S, Batut P, Chaisson M, Gingeras TR: STAR: ultrafast universal RNA-seq aligner. Bioinformatics 2013, 29(1):15-21.

86. Li B, Dewey CN: RSEM: accurate transcript quantification from RNA-Seq data with or without a reference genome. BMC bioinformatics 2011, 12:323.

87. Langfelder $\mathrm{P}$, Horvath S: WGCNA: an R package for weighted correlation network analysis. $B M C$ bioinformatics 2008, 9:559.

88. Smoot ME, Ono K, Ruscheinski J, Wang P-L, Ideker T: Cytoscape 2.8: new features for data integration and network visualization. Bioinformatics 2011, 27(3):431-432.

89. Huerta-Cepas J, Forslund K, Coelho LP, Szklarczyk D, Jensen LJ, von Mering C, Bork P: Fast GenomeWide Functional Annotation through Orthology Assignment by eggNOG-Mapper. Molecular biology and evolution 2017, 34(8):2115-2122.

90. Yu G, Wang LG, Han Y, He QY: clusterProfiler: an R package for comparing biological themes among gene clusters. Omics: a journal of integrative biology 2012, 16(5):284-287.

\section{Figures}



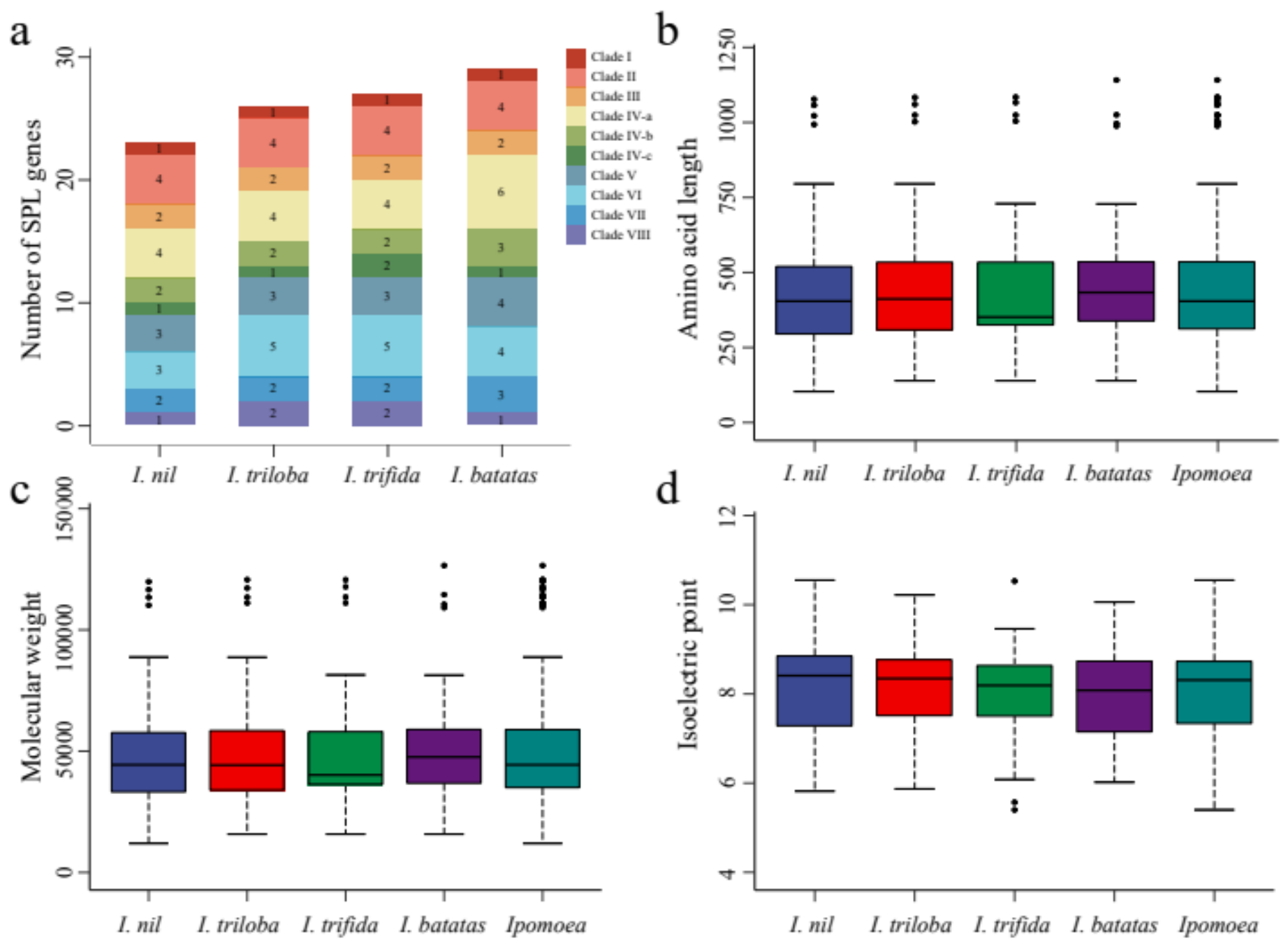

Figure 1

Comparison of SPL genes in four Ipomoea species (a) Comparison of the number and ratio of SPL genes in four Ipomoea species, the clades are displayed in different colors. (b-d) Comparison of SPL protein lengths, MWs and pls in four Ipomoea species, respectively. 


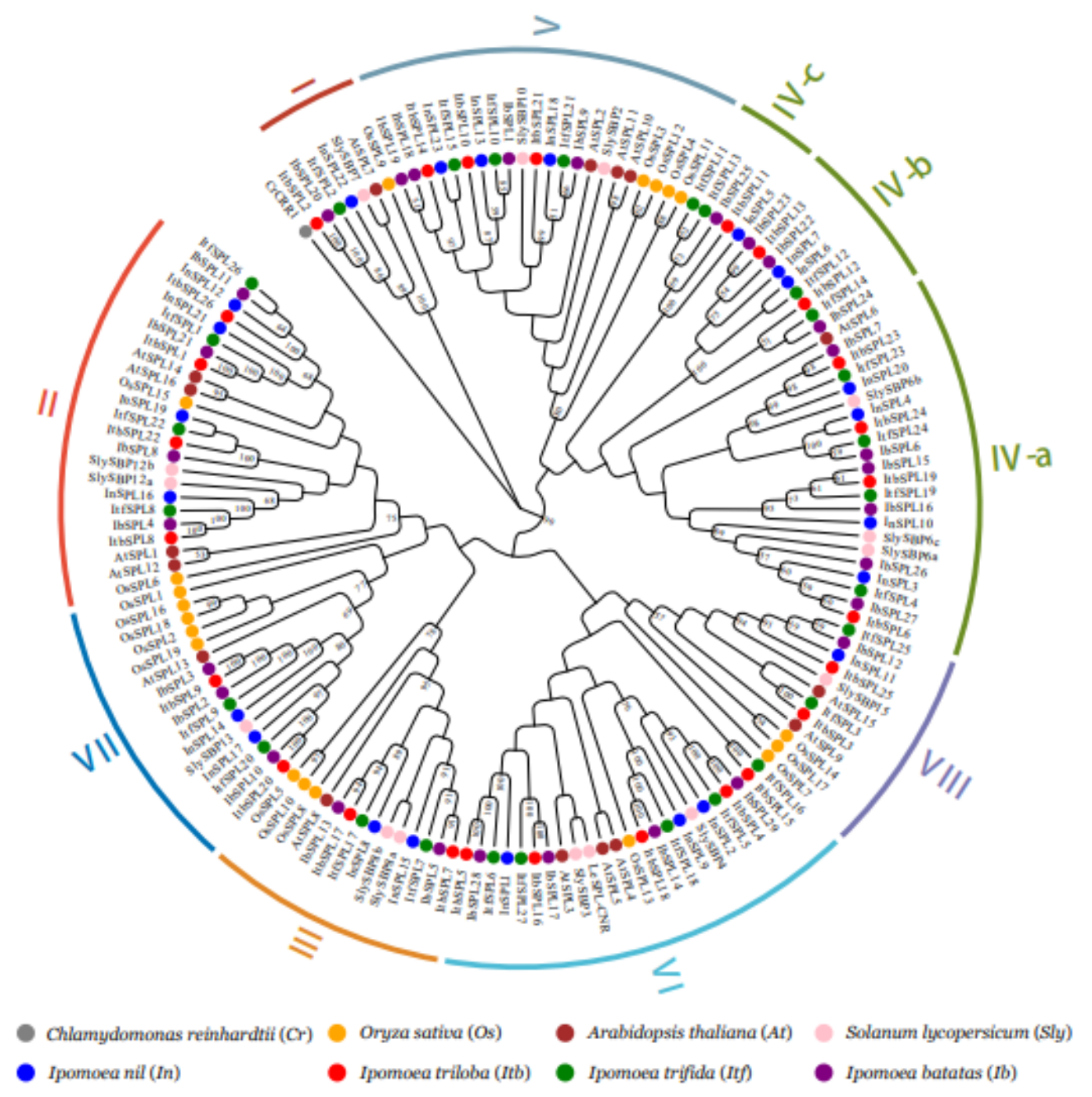

Figure 2

Phylogenetic analysis of SPL proteins from 4 Ipomoea species, C. reinhardtii, A. thaliana, S. lycopersicum, and O. sativa The rooted phylogenetic tree was constructed based on the conserved domain of 156 SPLs using neighbor-joining method with 1000 bootstrap replications. CrCRR1 protein from C. reinhardtii was used as an outgroup to root the tree. Numbers on the tree indicate bootstrap support (values $<50 \%$ not shown). The different-colored arcs indicate different clades of the SPLs. SPL members from the same species are marked with the same colors: Blue, I. nil; red, I. triloba; green, I. trifida; purple, I. batatas; grey, C. reinhardtii; orange, O. sativa; brown, A. thaliana; pink, S. lycopersicum. 

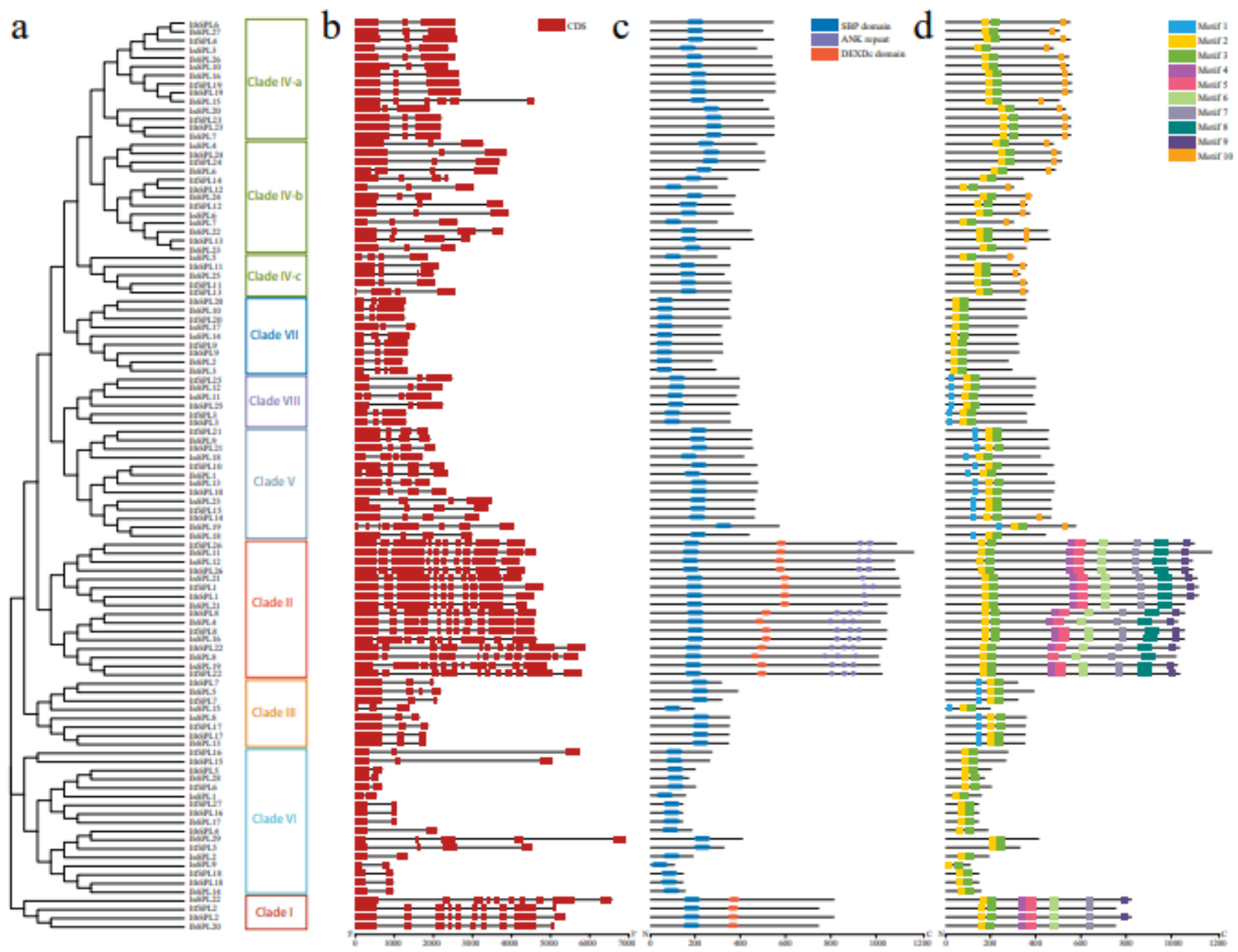

Figure 3

Phylogenetic relationships, gene structures, domain organizations, and motif compositions of SPL genes in Ipomoea species (a) Phylogenetic tree of 105 Ipomoea SPL proteins. (b) Intron-exon structures of Ipomoea SPL genes. Red box indicates coding sequence, and black line represents intron. (c) Domain organizations of Ipomoea SPL proteins. SBP domain, ANK repeat and DEXDc domain are exhibited in blue, purple, and orange boxes, respectively. (d) Motif compositions of Ipomoea SPL proteins. The ten motifs are displayed in different colored boxes. The length of genes or proteins can be estimated using the scale at the bottom. 

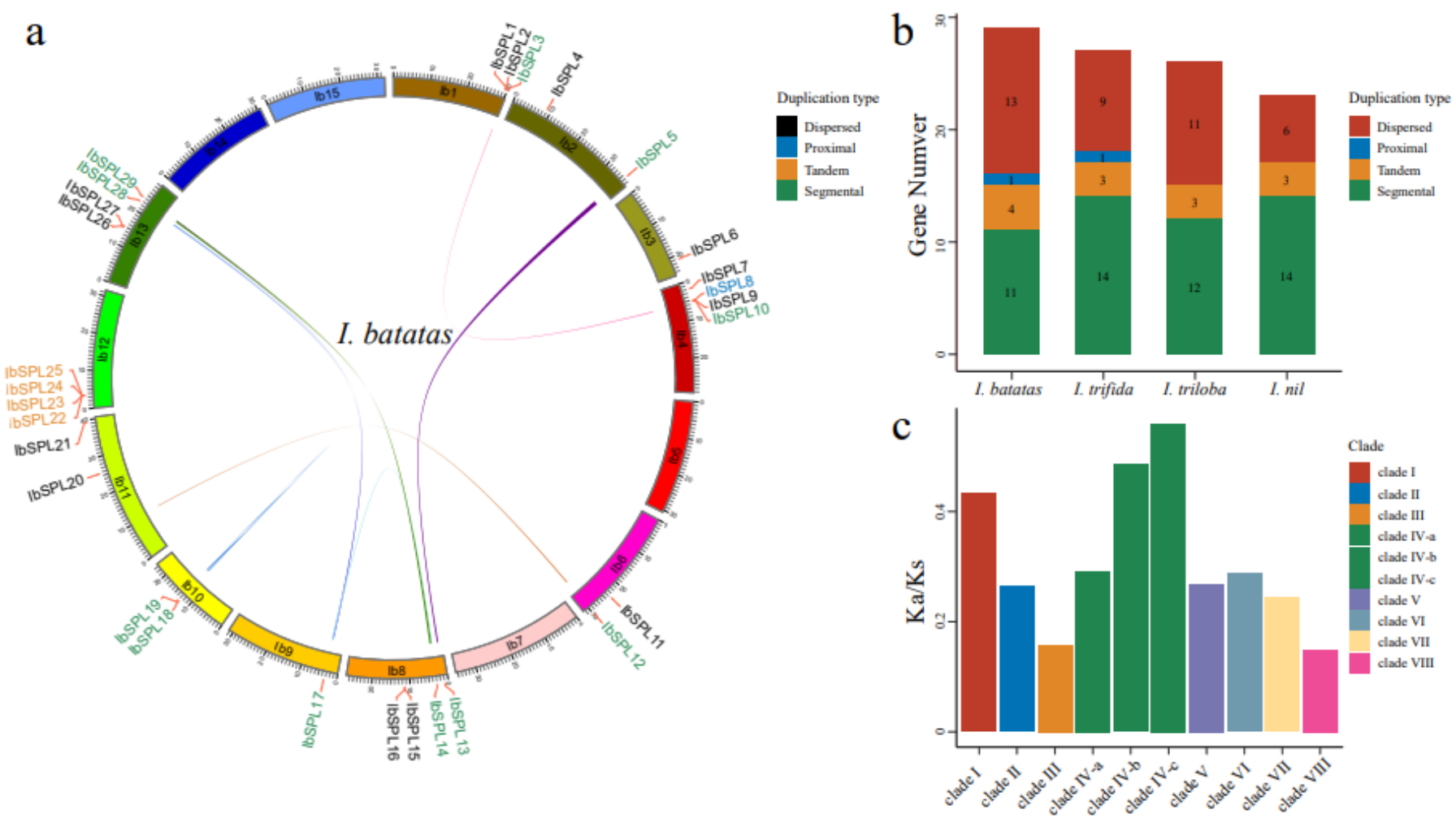

Figure 4

Syntenic relationships and selective pressure of Ipomoea SPLs (a) The colinear relationship among SPL genes in I. batatas. Colored lines indicate syntenic regions. IbSPL genes can be classified into four duplicated types, and marked as different colors. (b) The distribution of SPLs duplication modes in four Ipomoea species. (c) The mean Ka/Ks values in each clade. 
a

AthmiR156a

LG1 904913 904933+

LG6 $515391 \overline{9} \quad 5153939$ -

LG7 $2869406 \overline{9} 28694089-$

LG7 $28693363^{-} 28693383-$

LG8-8619326 $8619346-$

LG1 $\overline{2} \_145460 \overline{7} 1454627+$

LG13-22238208 22238228 -

LG15-17853788-17853808+ :

LG15_19018833_19018853-:

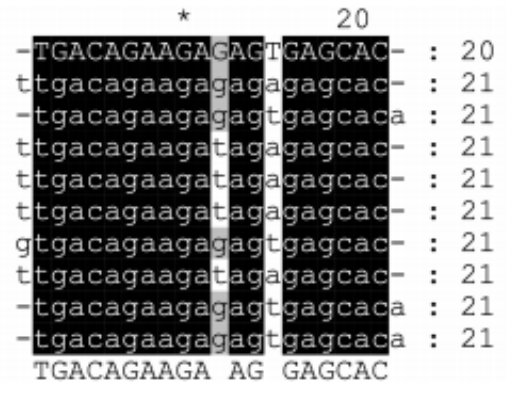

b

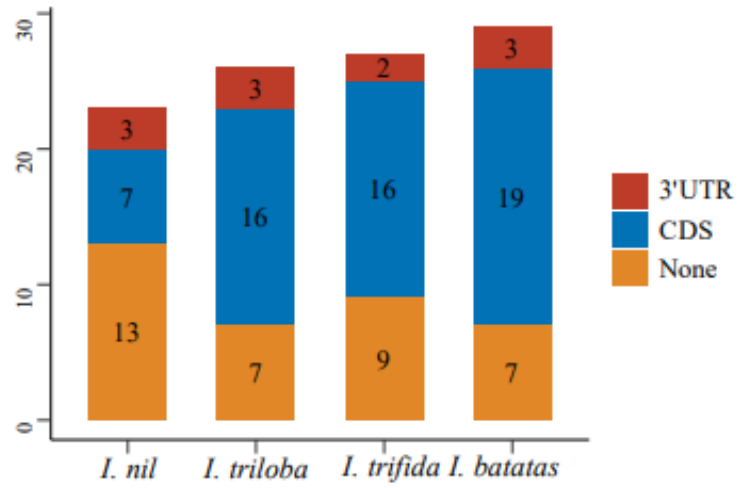

C

IbSPL9

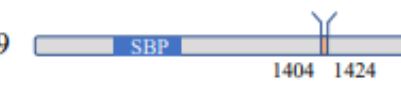

LG15_17853788_17853808+
3' ACACGAGUGAGAGAAGACAGU
$\vdots$
CAUGCUCUCUCUCUUCUGUCA

LG15_17853788_17853808+
3' ACACGAGUGAGAGAAGACAGU 5'
$\vdots \vdots|||||||||||||||||| \mid$
CAUGCUCUCUCUCUUCUGUCA

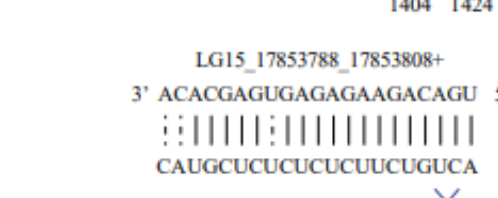

LG15_17853788_17853808+
3' ACACGAGUGAGAGAAGACAGU
$\vdots$
CAUGCUCUCUCUCUUCUGUCA

LG8_8619326_8619346-

' CacgagagauagahacaguU 5'

||||||||| |||||||||||

GUGCUCUCUCUCUUCUGUCAA

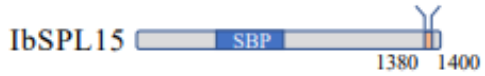
IbSPL16
LG6 5153919 51539393' ACACGagugagagadgacagu 5' i|||||| |||||||||||| CGUGCUCUCUCUCUUCUGUCA
LG1 904913 904933+

3' CacgagagagagangacaguU 5' |||||||||||||||||| GUGCUCUCUCUCUUCUGUCAA

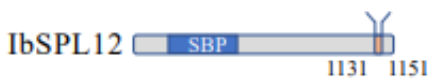

LG6 5153919 5153939-

3' ACACGagugagagaAgacAgU 5' 浇||||||||||||||| AUUGCUUACUCUCUUCUGUCA

\section{Figure 5}

miR156 target site of Ipomoea SPL genes (a) Multiple alignments of identified miR156 sequences. (b) Summary of miR156-targeted SPL genes in Ipomoea genus. (c) Diagram of IbSPL genes targeted by IbmiR156. The grey box indicates CDS region, the blue box represents SBP domain, and the red box indicates IbmiR156 target sites. 

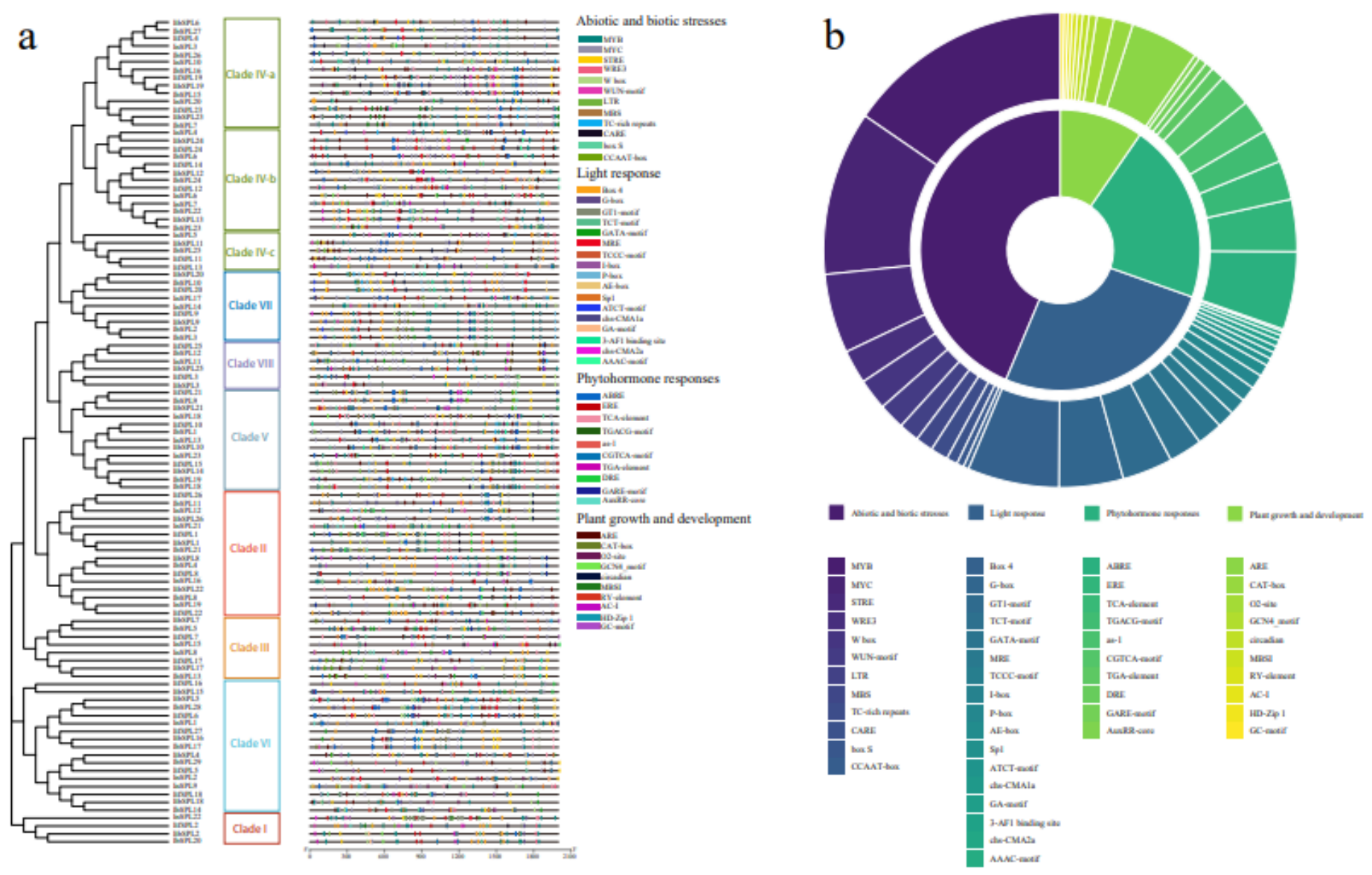

Figure 6

Investigation of cis-acting elements in Ipomoea SPL genes (a) Cis-acting elements in the promoter regions of all Ipomoea SPL genes. (b) Pie charts of cis-acting elements in all SPL genes. The colored boxes represent different categories or cis-acting elements. 

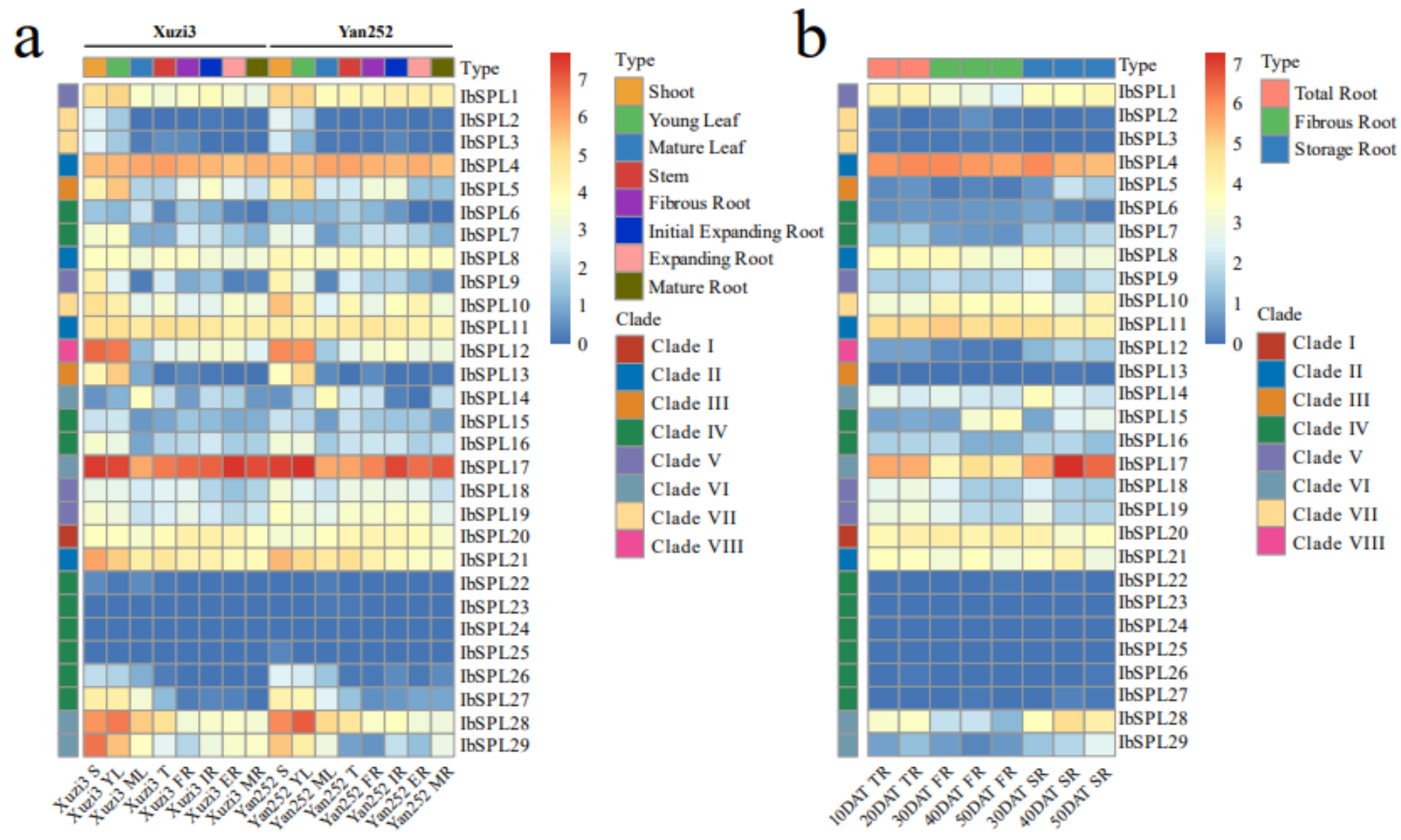

Figure 7

Expression profiles of IbSPL genes in different tissues and developmental stages by transcriptome analysis (a) Heatmap representing the expression patterns of IbSPL genes in 8 different tissues of two sweet potato cultivars. Xuzi3: sweet potato cultivar Xuzi3, Yan252: sweet potato cultivar Yan252; S: shoot, YL: young leaf, ML: mature leaf, T: stem, FR: fibrous root, IR: initial tuberous root, ER: expending storage root, MR: mature storage root. (b) Heatmap representing the expression patterns of IbSPL genes in eight different stages during root development. 10DAT_TR: undifferentiated root (10 days after transplanting), 20DAT_TR: undifferentiated root (20 days after transplanting), 30DAT_FR: fibrous root (30 days after transplanting), 40DAT40_FR: fibrous root (days after transplanting), 50DAT_FR: fibrous root (50 days after transplanting), 30DAT_SR: storage root (30 days after transplanting), 40DAT_SR: storage root (40 days after transplanting), 50DAT_SR: storage root (50 days after transplanting). Heatmap was generated based on $\log 2(\mathrm{FPKM}+1)$ values for each IbSPL gene. 

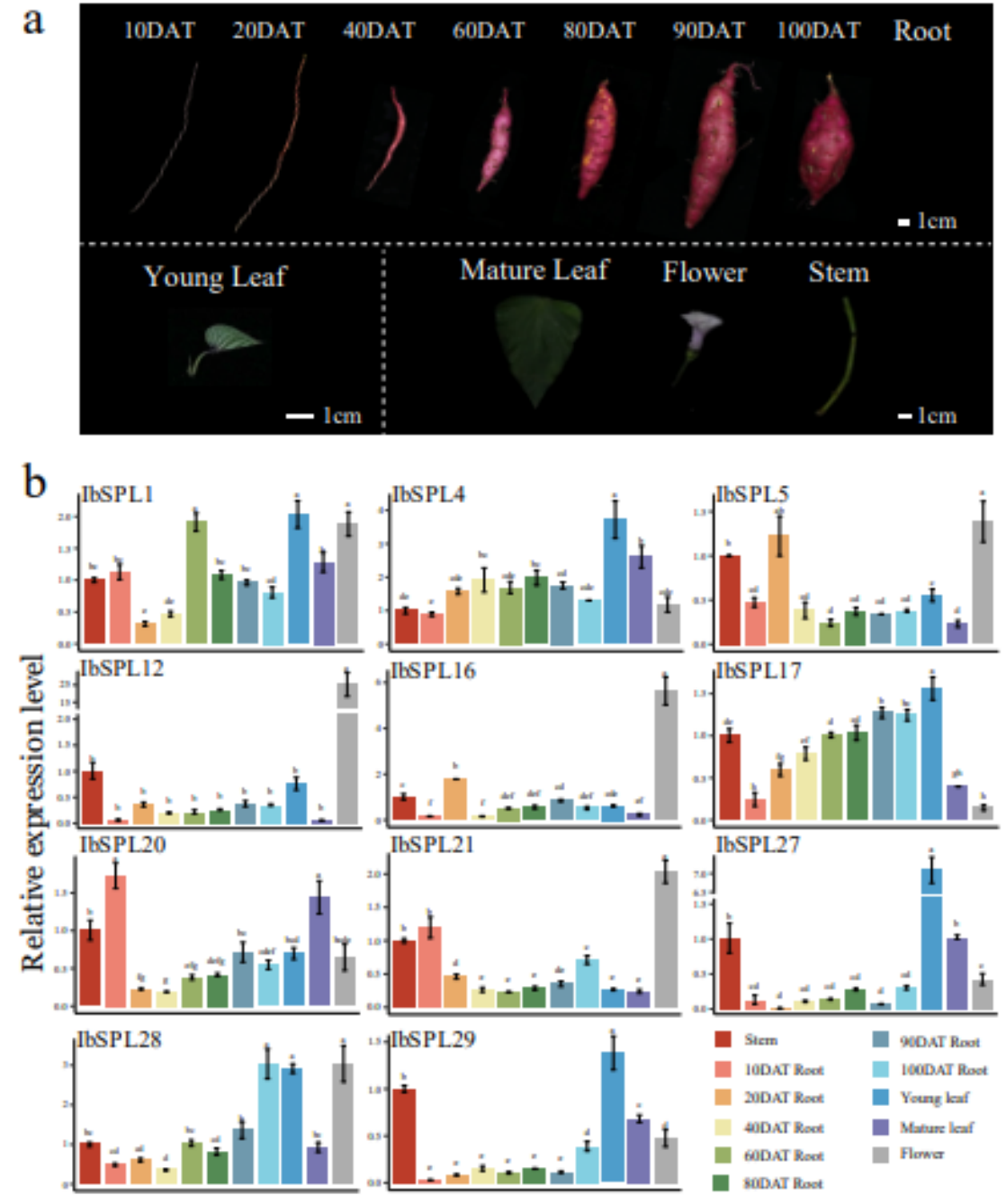

\section{Figure 8}

Expression profiles of $11 \mathrm{lbSPL}$ genes in different tissues and developmental stages by qRT-PCR (a) Different tissues from cultivar Xuyu34 were shown as pictures, including stem, leaf, flower and root. Black bar is scale of $1 \mathrm{~cm}$. (b) Expression patterns of $11 \mathrm{lbSPL}$ genes determined by qRT-PCR. The $x$-axis indicates different tissues. The $y$-axis represents the relative expression calculated by the $2-\Delta \Delta C T$ method. The error bars indicate the standard deviations of three biological replicates. Different letters indicate statistically significant difference at $p<0.05$. 

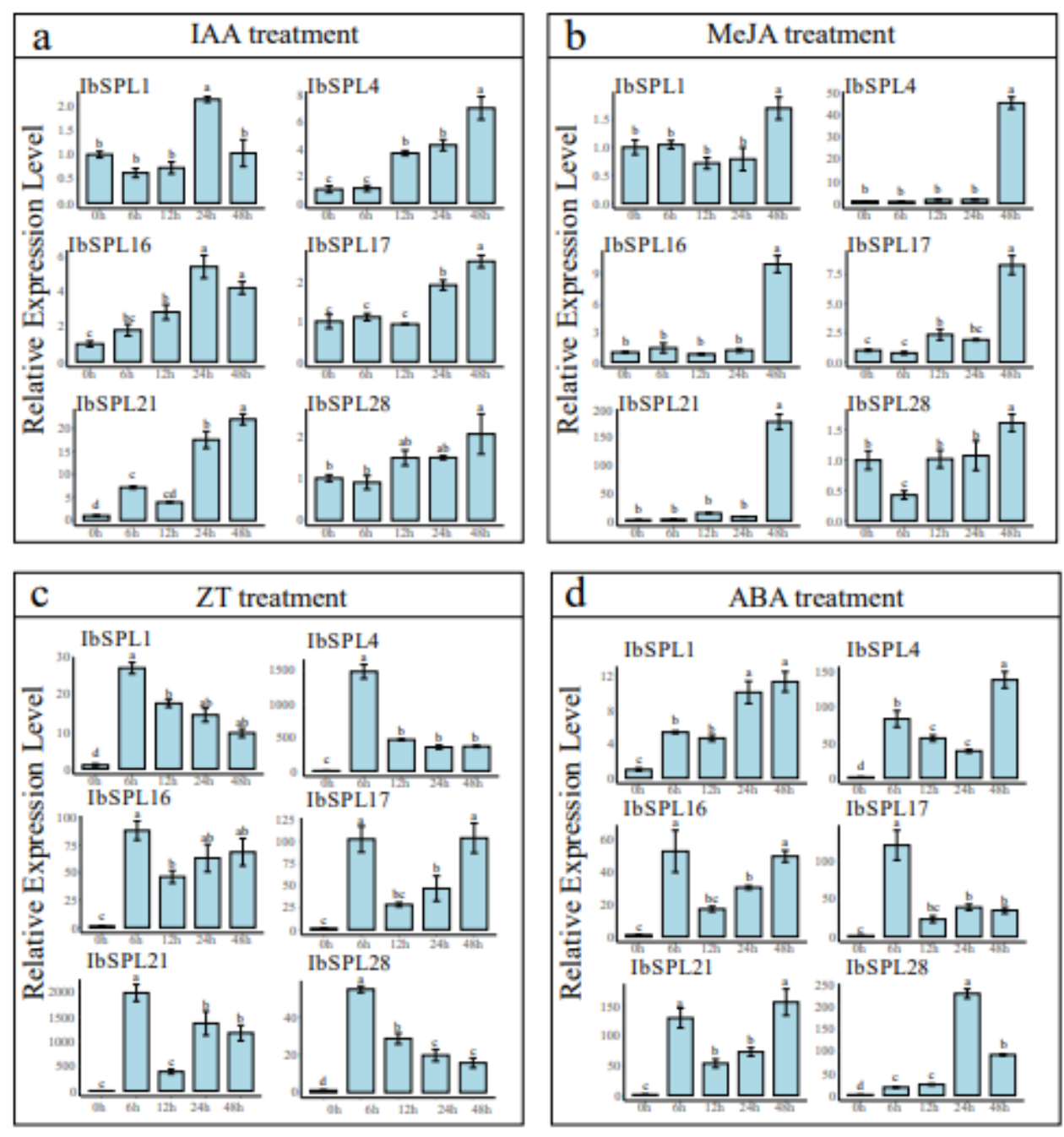

Figure 9

Expression patterns of $6 \mathrm{lbSPL}$ genes under phytohormone treatments (a) Expression patterns of $6 \mathrm{lbSPL}$ genes under IAA treatment. (b) Expression patterns of $6 \mathrm{lbSPL}$ genes under MeJA treatment. (c) Expression patterns of $6 \mathrm{lbSPL}$ genes under ZT treatment. (d) Expression patterns of $6 \mathrm{lbSPL}$ genes under ABA treatment. Samples were collected at $0,6,12,24$, and $48 \mathrm{~h}$ after treatment. The $\mathrm{x}$-axis indicates different samples. The $y$-axis represents the relative expression calculated by the $2-\Delta \Delta C T$ method. The error bars indicate the standard deviations of three biological replicates. Different letters indicate statistically significant difference at $p<0.05$. 
a

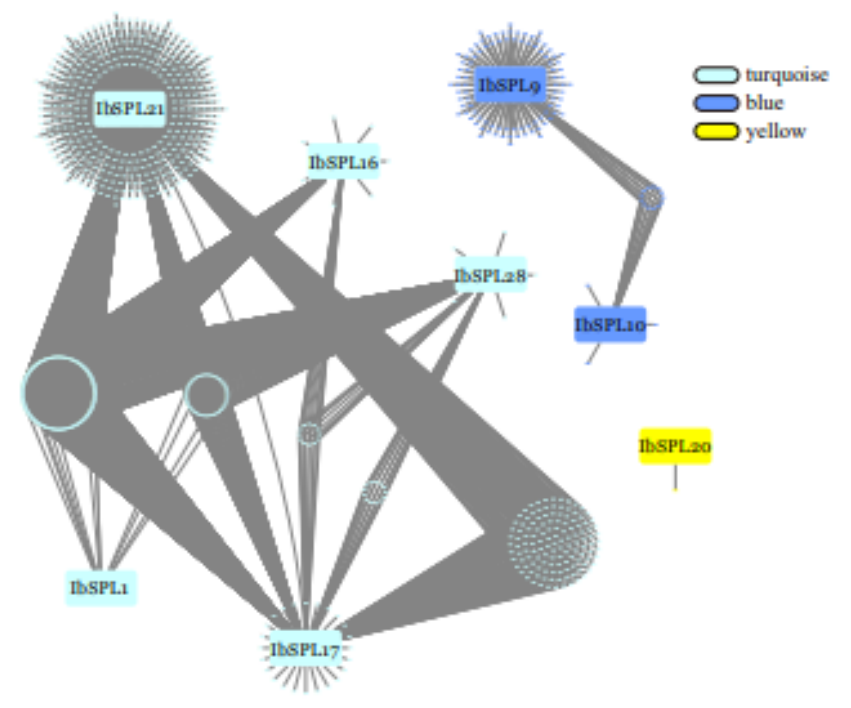

b

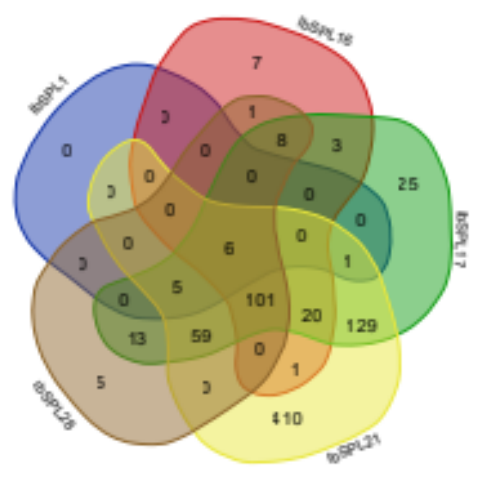

c

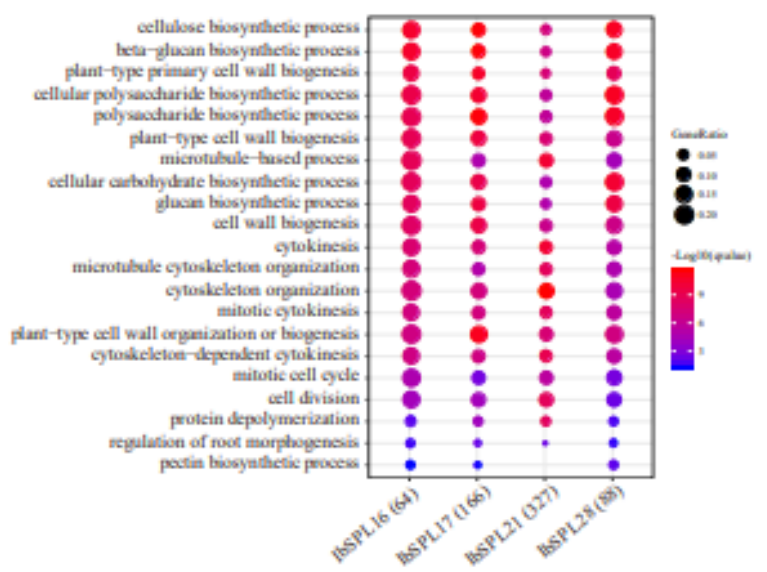

Figure 10

Regulatory sub-networks involving IbSPLs and other I. batatas genes in the storage root of sweet potato (a) Co-expression sub-network visualization for IbSPLs in the storage root of sweet potato. Only edges with weight above a threshold of 0.05 are displayed. (b) Venn diagram of the number of co-expressed genes for IbSPL1, IbSPL16, IbSPL17, IbSPL21, and IbSPL28 in the turquoise module. (c) G0 enrichment analysis of co-expressed genes of IbSPL16, IbSPL17, IbSPL21, and IbSPL28, respectively.

\section{Supplementary Files}

This is a list of supplementary files associated with this preprint. Click to download.

- SupplementaryTables.xls

- SupplementaryFigures.docx 